\title{
Comprehensive Analysis of the Expression and Prognosis of 12 Cytochrome P450s in Human Hepatocellular Carcinoma from the Perspective of Network Pharmacology
}

\section{Peng Jiang ( $\nabla$ jiangp@ustc.edu.cn )}

1 Department of Pharmacy, Division of Life Sciences and Medicine, The First Affiliated Hospital of USTC, University of Science and Technology of China https://orcid.org/0000-0001-8727-2663

\section{Qian Wang}

2 Department of Pharmacy, Anhui University of Chinese Medicine, Hefei, Anhui, 230012, China. 3 Institute of Pharmaceutics, Anhui Academy of Chinese Medicine, Hefei, Anhui, 230012, China. 4 Anhui Province Key Laboratory of Pharmaceutical Formulation Techno

\section{Yinghong Wang}

1 Department of Pharmacy, Division of Life Sciences and Medicine, The First Affiliated Hospital of USTC, University of Science and Technology of China

\section{Kai Li}

1 Department of Pharmacy, Division of Life Sciences and Medicine, The First Affiliated Hospital of USTC, University of Science and Technology of China

\section{Yancai Sun}

1 Department of Pharmacy, Division of Life Sciences and Medicine, The First Affiliated Hospital of USTC, University of Science and Technology of China

\section{Research}

Keywords: Cytochrome P450, Hepatocellular carcinoma, Bioinformatics, Network pharmacology, Sorafenib

Posted Date: August 19th, 2020

DOl: https://doi.org/10.21203/rs.3.rs-60063/v1

License: (c) (1) This work is licensed under a Creative Commons Attribution 4.0 International License. Read Full License 


\section{Abstract}

Background: Cytochrome P450s (CYPs) are pivotal drug metabolic enzymes and play a crucial part in the development and prognosis of hepatocellular carcinoma (HCC). Among various CYPs, 12 (CYP1A2, 27A1, $2 \mathrm{~A} 6,2 \mathrm{~A} 7,2 \mathrm{~B} 6,2 \mathrm{C} 8,2 \mathrm{C} 9,2 \mathrm{E} 1,2 \mathrm{~J} 2,3 \mathrm{~A} 4,3 \mathrm{~A} 5$, and $4 \mathrm{~A} 11$ ) are recognized as key therapeutic targets for $\mathrm{HCC}$ from the perspective of network pharmacology. However, the gene expression and prognosis of these 12 CYPs in HCC remain obscure.

Methods: In the current study, the mRNA expression of these 12 CYPs and survival of patients with HCC were investigated by mining data from ONCOMINE, Kaplan-Meier plotter, UALCAN, Human Protein Atlas, cBioPortal, STRING, and DAVID databases. Network pharmacology analysis of sorafenib for HCC treatment was conducted using data from Therapeutic Target Database, Drugbank, Swiss Target Prediction, STITCH, OncoDB.HCC, Liverome, STRING, and DAVID databases.

Results: Results showed that the mRNA expression levels of the 12 CYPs were markedly reduced in HCC and negatively correlated with tumor stages and grades (except for CYP3A5). The high expression level of nine CYPs, namely, CYP27A1, 2A6, 2A7, 2C8, 2C9, 2E1, 3A4, 3A5, and 4A11 was significantly correlated with favorable prognosis in patients with $\mathrm{HCC}$, indicating that they may serve as candidate prognostic biomarkers for HCC. Additionally, overexpression of CYP27A1, CYP2A7, CYP2B6, CYP2C9, and CYP3A5 was markedly correlated with favorable overall survival in HCC patients who received sorafenib therapy. Network pharmacology analysis of the administration of sorafenib for HCC treatment suggested that this drug may exert its antihepatocarcinoma effects by regulating the FoxO and ErbB signaling pathways.

Conclusion: Characterization of the expression and prognosis of 12 CYPs in HCC, as well as network pharmacology analysis of HCC treatment via sorafenib, may provide novel insights into the discovery of potential prognostic markers of and therapeutic targets in HCC.

\section{Background}

As the second leading cause of cancer-related deaths worldwide, hepatocellular carcinoma (HCC) is a malignant tumor with rising incidence and high mortality rate [1]. HCC is a complicated disease mainly induced by obesity, viral infection, aflatoxin exposure, alcohol abuse, and cirrhosis. However, its molecular features remain unclear. Furthermore, most patients with HCC are diagnosed at advanced stages at which point the only therapeutic option is systemic treatment using sorafenib. However, patients present varying responses to sorafenib, and many of them have to withdraw medication owing to sorafenib resistance and serious adverse effects [2]. Therefore, the molecular mechanisms of HCC initiation, development, and progression must be investigated. Moreover, an individualized HCC treatment using sorafenib should be developed to improve the overall survival of patients.

Network pharmacology, an emerging discipline based on systems biology, combines computer science and bioinformatics to construct biological networks of drugs, network targets, and diseases. This approach has been successfully applied in the study of pharmacological mechanisms and therapeutic 
target discovery [3]. A previous report stated that 566 HCC-related target genes were retrieved from two human liver cancer databases, namely, Liverome (http://liverome.kobic.re.kr/index.php) and OncoDB.HCC (http://oncodb.hcc.ibms.sinica.edu.tw). These HCC-related targets may provide novel insights into the pathogenesis, therapeutic effects, and prognosis of HCC. Among these targets, 12 cytochrome P450 enzymes (CYPs), namely, CYP1A2, CYP27A1, CYP2A6, CYP2A7, CYP2B6, CYP2C8, CYP2C9, CYP2E1, CYP2J2, CYP3A4, CYP3A5, and CYP4A11, are involved. CYPs, which are chiefly expressed in the liver, play a vital role in the metabolism of endogenous and xenobiotic substances, including antineoplastic drugs. A previous study reported that abnormal regulation of cytochrome P450 expression and activities in HCC have implications to personalized treatment [4-6]. Moreover, CYP2C8 [7], CYP2C9 [8], the CYP3A subfamily [9], and CYP4A11 [10] are reportedly closely related to HCC prognosis and survival. Therefore, the expression and prognosis of key CYP family members in HCC must be clarified to reveal the molecular mechanisms underlying HCC pathogenesis and provide novel insights into personalized HCC treatment via sorafenib administration. To the best of our knowledge, no one has conducted yet a network pharmacology analysis of HCC treatment using sorafenib. Network pharmacology offers a novel approach for unveiling new therapeutic targets and understanding the pharmacological mechanisms of sorafenib action in HCC.

At least 36 CYP family members have been identified in humans [11]. Previous studies largely focused on individual or several markedly expressed CYP proteins between normal and end-stage liver disease in determining prognosis [6]. Owing to this limitation of previous studies, we conducted a comprehensive analysis of the expression and prognosis of $12 \mathrm{CYPs}$ in HCC from the perspective of network pharmacology. Moreover, we performed a network pharmacology analysis of sorafenib administration for HCC treatment.

\section{Methods}

\section{ONCOMINE database}

The ONCOMINE database [12] (https://www.oncomine.org/resource/login.html), an integrated cancer microarray online database, was utilized to characterize the transcription levels of 12 CYPs between various cancers and their corresponding normal control tissues. Differences in mRNA expression levels were compared using Student's $t$ test. The screening conditions were set as follows [13]: data type: mRNA, $p$ value: $<0.01$, fold change: $\geq 1.5$.

\section{UALCAN}

UALCAN [14] (http://ualcan.path.uab.edu/) is a comprehensive and interactive web portal built on the transcriptome sequencing and clinical data of 31 cancer types from The Cancer Genome Atlas (TCGA) project. This platform characterizes transcriptional gene expression level along with clinicopathologic features between tumor and normal samples on patient survival. In the present study, the mRNA expressions of 12 CYPs family members in normal and certain HCC clinicopathological parameters (i.e., primary tumor, individual cancer stages and tumor grades) were analyzed using UALCAN. Differences in 
transcriptional expression difference between groups were compared using Student's $t$ test, and the criterion of statistical significance was $p<0.05$.

\section{Human Protein Atlas}

The Human Protein Atlas [15] (https://www.proteinatlas.org) is a database devoted to investigating human protein distribution and expression information. In building the Human Protein Atlas database, immunohistochemistry was performed to display the protein expression data of numerous different normal tissues and tumor pathological tissues. This database provides high-quality immunohistochemical images. In the present study, the protein expressions of 12 CYP family members between normal and HCC tissues were intuitively compared via immunohistochemical staining with a corresponding specific antibody.

\section{Kaplan-Meier plotter}

Kaplan-Meier plotter [16] (http://kmplot.com/analysis/) is an online database for discovering and validating survival biomarkers. This database can evaluate the effects of $54 \mathrm{k}$ genes on survival in 21 cancer types, including HCC. Multiple genes of the 12 CYP family members were searched in KaplanMeier plotter to draw survival curves on the basis of 364 patients with HCC. Among these patients, 29 received systemic treatment using sorafenib. The survival curves of these patients were also generated to explore the relationship between sorafenib treatment and the expression of the $12 \mathrm{CYPs}$. Median values of mRNA expression in both high-and low-expression groups, hazard ratio (HR), and log rank $p$ value were harvested from the database.

\section{TCGA database and cBioPortal}

TCGA [17] is a comprehensive database that contains cancer genomic profiles of 30 kinds of human cancers. The liver HCC (TCGA, PanCancer Atlas) dataset, including 372 cases, was chosen to analyze the 12 CYP members in cBioPortal (https://www.cbioportal.org/) $[18,19]$. cBioPortal is an online database for exploring and visualizing multidimensional tumor genomics data. The criteria of genomic profiles were set as follows [13]: mutations, mRNA expression z-scores (RNA-seq V2 RSEM), and putative copy-number alterations from GISTIC. Genetic mutations of CYPs, along with overall survival (OS) and disease-free survival (DFS) of patients with HCC, were obtained from this platform.

\section{Protein-protein interaction network construction, Gene Ontology, and Kyoto Encyclopedia of Genes and Genomes analysis}

Multiple proteins of the 12 CYPs were inputted into the STRING database [20] (https://string-db.org/) to build a protein-protein (PPI) network. The STRING database aims to collect and integrate knowledge of all functional interactions between expressed proteins. The parameters were set as follows: the highest confidence score was $>0.9$, and the maximum number of interactors was $\leq 50$. The results were imported into Cytoscape software (ver. 3.7.2) for visualization and further analysis. Gene Ontology (GO) and Kyoto Encyclopedia of Genes and Genomes (KEGG) pathway analyses of the 12 CYPs were 
performed using the Database for Annotation, Visualization, and Integrated Discovery (DAVID) (http://david.abcc.ncifcrf.gov) [21]. DAVID is a comprehensive functional annotation database for exploring the biological significance of multiple genes. The results of GO and KEGG pathway analyses were displayed using GraphPad Prism software (ver. 7.0).

\section{Network pharmacology analysis of sorafenib administration for HCC treatment}

The human protein targets of sorafenib were retrieved from the Therapeutic Target Database (http://db.idrblab.net/ttd/) [22], Drugbank database (https://www.drugbank.ca/) [23], Swiss Target Prediction database (http://www.swisstargetprediction.ch/) [24], and STITCH database (http://stitch.embl.de/). HCC-related targets were obtained from the two hepatic carcinoma-related databases OncoDB.HCC (http://oncodb.hcc.ibms.sinica.edu.tw) [25] and Liverome (http://liverome.kobic.re.kr/index.php) [26]. The human protein targets of sorafenib were then mapped with HCC-related genes to seek for candidate targets of sorafenib for HCC treatment. The candidate targets were imported into the STRING database and visualized using Cytoscape software. GO and KEGG pathway analyses of the candidate targets were conducted using the DAVID database.

\section{Results}

\section{Low CYP expression in patients with HCC}

The transcription and protein levels of the 12 CYPs members were evaluated using the ONCOMINE, UALCAN, and Human Protein Atlas databases. As shown in Figure 1, the mRNA expression levels of the 12 CYPs were significantly downregulated in HCC compared with those in normal liver according to the ONCOMINE database. The detailed results of $p$ value and fold change are shown in Table 1. The $p$ value ranged from -1.512 to -63.196 , whereas fold change ranged from 0.001 to $1.5 \mathrm{E}-131$. For example, the mRNA expression level of CYP1A2 in HCC remarkably decreased in the three datasets compared with that in normal liver. In the Roessler liver dataset, the expression of CYP1A2 in HCC was low compared with that in normal liver, with a fold change of $-63.196(p=4.58 \mathrm{E}-21)$. In Roessler liver 2, the mRNA expression level of CYP1A2 in HCC decreased by 18.872-fold $(p=1.5 \mathrm{E}-131)$. In Wurmbach liver, the mRNA expression level of CYP1A2 in HCC samples decreased by 23.814-fold ( $p=1.69 \mathrm{E}-09)$. The mRNA expression levels of the other CYPs in HCC tissues were substantially lower in at least two datasets (Table 1). The transcriptional levels of the 12 CYP members were further determined in the UALCAN database, which is quite different from the ONCOMINE database. With the exception of CYP2A7 and CYP3A5, the mRNA expression levels of the CYP members considerably decreased in HCC samples compared in normal liver samples $(p<0.001)$. The protein expression levels of the 12 CYP members in HCC were explored using the Human Protein Atlas database. Most immunohistochemical staining of CYP members can be found in the database, except for that of CYP2J2, CYP3A5, and CYP4A11. The protein expression levels of CYP1A2, CYP2A6, CYP2A7, CYP2B6, CYP2C8, CYP2C9, and CYP2E1 were high in normal liver tissues, whereas these proteins were not detected in HCC tissues. Additionally, the protein expression level of CYP27A1 was medium and that of CYP3A4 was high in normal liver tissues, but no proteins were 
detected or their expression levels were low in HCC tissues. Based on the results of analyses using these three databases, the transcription and protein expression levels of the 12 CYP members were low in patients with HCC patients.

Table 1. The significant changes of CYPs expression in transcription level between hepatocellular carcinoma and normal liver tissues (ONCOMINE database) 


\begin{tabular}{|c|c|c|c|c|c|}
\hline & Types of HCC VS. Liver & $\begin{array}{l}\text { Fold } \\
\text { Change }\end{array}$ & $P$ value & t-test & Reference \\
\hline \multirow[t]{3}{*}{ CYP1A2 } & $\begin{array}{l}\text { Hepatocellular } \\
\text { Carcinoma }\end{array}$ & -63.196 & $4.58 \mathrm{E}-21$ & -22.801 & Roessler Liver[27] \\
\hline & $\begin{array}{l}\text { Hepatocellular } \\
\text { Carcinoma }\end{array}$ & -18.872 & $\begin{array}{l}1.50 \mathrm{E}- \\
131\end{array}$ & -35.521 & Roessler Liver 2[27] \\
\hline & $\begin{array}{l}\text { Hepatocellular } \\
\text { Carcinoma }\end{array}$ & -23.814 & 1.69E-09 & -8.942 & $\begin{array}{l}\text { Wurmbach } \\
\text { Liver[28] }\end{array}$ \\
\hline \multirow[t]{2}{*}{ CYP27A1 } & $\begin{array}{l}\text { Hepatocellular } \\
\text { Carcinoma }\end{array}$ & -1.936 & $2.21 \mathrm{E}-26$ & -11.77 & Roessler Liver 2[27] \\
\hline & $\begin{array}{l}\text { Hepatocellular } \\
\text { Carcinoma }\end{array}$ & -2.345 & 7.42E-05 & -4.467 & Roessler Liver[27] \\
\hline \multirow[t]{4}{*}{ CYP2A6 } & $\begin{array}{l}\text { Hepatocellular } \\
\text { Carcinoma }\end{array}$ & -11.335 & 7.21E-72 & -23.859 & Roessler Liver 2[27] \\
\hline & $\begin{array}{l}\text { Hepatocellular } \\
\text { Carcinoma }\end{array}$ & -6.534 & $6.56 \mathrm{E}-15$ & -8.617 & Chen Liver[29] \\
\hline & $\begin{array}{l}\text { Hepatocellular } \\
\text { Carcinoma }\end{array}$ & -7.586 & 1.75E-08 & -7.611 & Roessler Liver[27] \\
\hline & $\begin{array}{l}\text { Hepatocellular } \\
\text { Carcinoma }\end{array}$ & -9.301 & 9.69E-04 & -3.563 & $\begin{array}{l}\text { Wurmbach } \\
\text { Liver[28] }\end{array}$ \\
\hline \multirow[t]{3}{*}{ CYP2A7 } & $\begin{array}{l}\text { Hepatocellular } \\
\text { Carcinoma }\end{array}$ & -6.674 & 5.03E-79 & -25.133 & Roessler Liver 2[27] \\
\hline & $\begin{array}{l}\text { Hepatocellular } \\
\text { Carcinoma }\end{array}$ & -5.337 & $6.86 \mathrm{E}-10$ & -8.241 & Roessler Liver[27] \\
\hline & $\begin{array}{l}\text { Hepatocellular } \\
\text { Carcinoma }\end{array}$ & -9.49 & $6.55 \mathrm{E}-04$ & -3.733 & $\begin{array}{l}\text { Wurmbach } \\
\text { Liver[28] }\end{array}$ \\
\hline \multirow[t]{4}{*}{ CYP2B6 } & $\begin{array}{l}\text { Hepatocellular } \\
\text { Carcinoma }\end{array}$ & -13.569 & 7.56E-18 & -14.541 & Roessler Liver[27] \\
\hline & $\begin{array}{l}\text { Hepatocellular } \\
\text { Carcinoma }\end{array}$ & -10.802 & $\begin{array}{l}3.59 \mathrm{E}- \\
100\end{array}$ & -29.077 & Roessler Liver 2[27] \\
\hline & $\begin{array}{l}\text { Hepatocellular } \\
\text { Carcinoma }\end{array}$ & -2.955 & $9.51 \mathrm{E}-27$ & -12.67 & Chen Liver[29] \\
\hline & $\begin{array}{l}\text { Hepatocellular } \\
\text { Carcinoma }\end{array}$ & -7.529 & 1.15E-04 & -4.63 & $\begin{array}{l}\text { Wurmbach } \\
\text { Liver[28] }\end{array}$ \\
\hline \multirow[t]{2}{*}{ CYP2C8 } & $\begin{array}{l}\text { Hepatocellular } \\
\text { Carcinoma }\end{array}$ & -5.766 & 2.32E-20 & -10.515 & Chen Liver[29] \\
\hline & $\begin{array}{l}\text { Hepatocellular } \\
\text { Carcinoma }\end{array}$ & -3.561 & 2.69E-05 & -5.393 & $\begin{array}{l}\text { Wurmbach } \\
\text { Liver[28] }\end{array}$ \\
\hline CYP2C9 & Hepatocellular & -4.874 & $2.89 \mathrm{E}-95$ & -27.301 & Roessler Liver 2[27] \\
\hline
\end{tabular}


Carcinoma

Hepatocellular

$-5.164$

2.15E-13

$-10.51$

Roessler Liver[27]

Carcinoma

Hepatocellular

$-5.606$

1.33E-19

$-10.216$

Chen Liver[29]

Carcinoma

Hepatocellular

$-4.438$

3.38E-08

$-6.534$

Wurmbach

Carcinoma

$-1.512$

2.06E-06

-5.172 Mas Liver[30]

CYP2E1 Hepatocellular

Carcinoma

Hepatocellular

$-8.697$

$3.46 \mathrm{E}-38$

$-15.287$

Roessler Liver 2[27]

Carcinoma

Hepatocellular

Carcinoma

$-3.825$

2.48E-05

$-4.613$

Wurmbach

Liver[28]

CYP2J2 Hepatocellular

Carcinoma

$-3.11$

1.16E-49

$-17.655$

Roessler Liver 2[27]

Hepatocellular

Carcinoma

$-2.112$

1.50E-11

$-7.105$

Chen Liver[29]

Hepatocellular

Carcinoma

$-1.982$

2.05E-05

$-4.897$

Wurmbach

Liver[28]

Hepatocellular

$-3.209$

3.54E-06 -5.653 Roessler Liver[27]

Carcinoma

CYP3A4 Hepatocellular

Carcinoma

$-4.23$

1.27E-12

$-11.406$

Roessler Liver[27]

Hepatocellular

$-7.094$

3.85E-71

$-23.668$

Roessler Liver 2[27]

Carcinoma

Hepatocellular

$-2.53$

3.17E-10

$-6.543$

Chen Liver[29]

Carcinoma

CYP3A5 Hepatocellular

Carcinoma

$-2.771$

4.63E-40

$-14.932$

Roessler Liver 2[27]

Hepatocellular

$-2.516$

8.02E-07

$-5.918$

Roessler Liver[27]

Carcinoma

Hepatocellular

$-1.809$

5.67E-08

$-5.531$

Chen Liver[29]

Carcinoma

$-5.733$

2.29E-80

$-24.804$

Roessler Liver 2[27]

Carcinoma

Hepatocellular

Carcinoma

$-3.806$

1.82E-09

$-7.533$

Roessler Liver[27]

Hepatocellular

$-2.655$

1.91E-08

$-5.769$

Chen Liver[29]

Carcinoma 
CYP: cytochrome P450, HCC: hepatocellular carcinoma

\section{Relationship between mRNA expressionlevels of the 12 CYP members and clinicopathological parameters of patients with HCC}

Using the UALCAN database (http://ualcan.path.uab.edu), we compared the mRNA expression levels of the 12 CYP members in normal liver tissues and HCC tissues at specific cancer stages and tumor grades. Except for that of CYP3A5, the mRNA expression levels of the CYP members were negatively correlated with individual cancer stages (Figure 4). Patients with HCC at advanced stages were presented lower mRNA expression levels of the CYPs than those with HCC at early stages. The mRNA expression levels of CYP 1A2, 2A7, 2C9, 2E1, and 3A4 were the lowest at stage 4. By comparison, the mRNA expression levels of CYP27A1, 2A6, 2B6, 2C8, 2J2, and $4 \mathrm{~A} 11$ were the lowest at stage 3 . Given that only six patients had stage $4 \mathrm{HCC}$, the mRNA expression levels of CYP 27A1, 2A6, 2B6, 2C8, 2J2, and 4A11 between stages 4 and 3 did not considerably differ. In addition, only the mRNA expression level of CYP3A5 significantly varied between stages 1 and 3. Similarly, the mRNA expression levels of the 12 CYP members (except for that of CYP2A7 and CYP3A5) negatively correlated with tumor grades (Figure 5), indicating that the mRNA expression levels of the CYP members decreased as tumor grades increased. The mRNA expression levels of CYP2A7 and CYP3A5 did not substantially differ between normal and different grades. These findings indicated that the mRNA expression levels of the $12 \mathrm{CYP}$ members strongly associated with the clinicopathological parameters of patients with HCC.

\section{Association of mRNA expression levels of the 12 CYP members and prognosis of patients with HCC}

The Kaplan-Meier plotter database (http://kmplot.com/analysis/) was utilized to determine the relationship between the mRNA expression levels of the 12 CYP members and the survival of patients with HCC. The high mRNA expression levels of CYP27A1 (HR = 0.42, P = 4.1E-07), CYP2A6 (HR = 0.54, $P=$ 7E-04), CYP2A7 (HR= 0.57, P = 0.0016), CYP2C8 (HR=0.54, P = 0.00048), CYP2C9 (HR=0.42, P = 3.2E07), CYP2E1 (HR = 0.57, $P=0.0037)$, CYP3A4 ( $H R=0.57, P=0.0017)$, CYP3A5 $(H R=0.43, P=1.4 E-05)$, and CYP4A11 $(H R=0.59, P=0.0025)$ were significantly correlated with long overall survival of patients with HCC. However, the differences in the mRNA expression levels of CYP1A2 (HR $=0.71, P=0.074)$, CYP2B6 $(\mathrm{HR}=0.71, \mathrm{P}=0.061)$, and CYP2J2 $(\mathrm{HR}=0.75, \mathrm{P}=0.11)$ and survival of patients with $\mathrm{HCC}$ were not significant. Thus, the mRNA expression levels of CYP27A1, CYP2A6, CYP2A7, CYP2C8, CYP2C9, CYP2E1, CYP3A4, CYP3A5, and CYP4A11 were significantly correlated with the OS of patients with HCC. These CYPs may be developed as valuable biomarkers to predict the prognosis of patients with HCC.

Among the 364 patients with HCC found in the Kaplan-Meier plotter database, 29 received sorafenib for HCC treatment. The relationship between the mRNA expression levels of the 12 CYPs and OS of the 29 patients with HCC was explored. As shown in Figure 7, only the high mRNA expression levels of CYP27A1, CYP2A7, CYP2B6, CYP2C9, and CYP3A5 were significantly correlated with favorable OS in the 29 patients 
with HCC $(p<0.05)$. Notably, the mRNA expression level of CYP2B6 $(H R=0.14, P=0.00076)$ was markedly related to long OS of the 29 patients. This result was inconsistent with that of CYP2B6 (HR = $0.71, P=0.061)$ in 364 patients with HCC. These results suggested that the mRNA expression level of CYP2B6 may be developed into a unique biomarker to predict the prognosis of patients with HCC who received sorafenib therapy.

\section{Genetic alterations in the 12 CYP members and their relationship with OS and DFS of patients with HCC}

The cBioPortal (https://www.cbioportal.org/) database was used to explore genetic mutations and their relevance to OS and DFS of patients with HCC. The genes of the 12 CYP members were altered in 144 out of 366 queried patients at a rate of $39 \%$ (Figure $8 \mathrm{a}$ ). The rate of genetic alteration of individual CYP members varied from $5 \%$ to $9 \%$. Moreover, no significant correlation was observed between genetic alteration in the 12 CYP members and OS (Figure 8b, Logrank $p=0.0955$ ) or DFS (Figure 8c, Logrank $p=$ 0.652). Thus, the genetic alterations in the $12 \mathrm{CYP}$ members did not seem to remarkably influence the prognosis of patients with HCC.

\section{PPI network construction of the 12 CYP members and analysis of their GO and KEGG pathways in patients with HCC}

The PPI network of the 12 CYP members and 50 target genes that they closely interact with was analyzed using the STRING database (https://string-db.org/). The CYP1A1 (degree = 24), AOX1 (degree = 18), CYP2C19 (degree $=15)$, CYP2D6 (degree $=13)$, and EPHX1 (degree $=12)$ of these 50 genes were significantly related to the 12 CYP members in the PPI network (Figure 9a). Subsequently, the GO and KEGG pathways of the 12 CYP members and 50 target genes that they closely interact with were analyzed using the DAVID database (http://david.abcc.ncifcrf.gov). The top 10 terms of GO and KEGG analysis are displayed in Figure 9. As shown in Figures 9b-9d, the biological processes strongly concerned with alterations in the 12 CYPs were xenobiotic metabolic process (GO: 0006805), drug metabolic process (GO: 0017144), oxidation-reduction process (GO: 0055114), epoxygenase P450 pathway (GO: 0019373), and steroid metabolic process (GO: 0008202). The cellular components substantially regulated by alterations in the 12 CYPs were organelle membrane (GO: 0031090), endoplasmic reticulum membrane (GO: 0005789), intracellular membrane-bounded organelle (GO: 0043231), endoplasmic reticulum (GO: 0005783), and integral component of membrane (G0: 0016021). The molecular functions notably influenced by the 12 CYP members were heme binding (GO: 0020037), monooxygenase activity (GO: 0004497), iron ion binding (GO: 0005506), steroid hydroxylase activity (GO: 0008395), and oxidoreductase activity, acting on paired donors, with incorporation or reduction of molecular oxygen (GO: 0016705). KEGG analysis was conducted to explore the altered pathways related to the 12 CYP members and their neighboring genes. As depicted in Figure $9 \mathrm{e}$, the pathways chemical carcinogenesis (hsa05204), drug metabolism - cytochrome P450 (hsa00982), metabolism of xenobiotics by cytochrome P450 (hsa00980), retinol metabolism (hsa00830), and metabolic pathways (hsa01100) were involved in the functions of the $12 \mathrm{CYP}$ and the target genes they closely interact with in HCC.

\section{Network pharmacology analysis of sorafenib administration for HCC treatment}


The flowchart of network pharmacology analysis of sorafenib administration for HCC treatment is shown in Figure 10. Four human protein targets and 10 human protein targets of sorafenib were obtained from the Therapeutic Target Database (http://db.idrblab.net/ttd/) and the Drugbank database (https://www.drugbank.ca/), respectively. Sorafenib acted as a substrate or inhibitor for 11 metabolic enzymes and six drug transporters that were regarded as targets of sorafenib. Afterward, 100 human targets (probability $\geq 0.9$ ) and 20 targets (confident $\geq 0.9$ ) were predicted from the Swiss Target Prediction database (http://www.swisstargetprediction.ch/) and the Stitch database (http://stitch.embl.de/), respectively. After removing the overlaps, a total of 123 protein targets (28 identifiable targets and 95 predictive targets) were obtained for further analysis (Additional file 1). A previous study [31] screened 566 HCC-related genes from both the OncoDB.HCC and Liverome databases (Additional file 2). Subsequently, human targets of sorafenib were mapped with HCC-related targets, and 25 targets (12 validated targets and 13 predicted targets) were harvested as candidate targets of sorafenib for HCC treatment (Additional file 3).

The PPI network of the 25 candidate targets of sorafenib was analyzed using the String database. Results showed that CCNA2 (degree $=24)$, SRC $($ degree $=23)$, MAPK1 $($ degree $=22)$, MAPK3 (degree = 21 ), AURKA (degree $=18$ ), and EGFR (degree $=16$ ) were the key potential targets in the network (Figure 11a). In addition, a group of candidate target CYPs, namely, CYP1A2, CYP2B6, CYP2C8, CYP2C9, CYP3A4, and CYP3A5, was included. The GO and KEGG pathways of the 25 candidate targets and their 50 closely related genes were analyzed using the David database (Figures $11 \mathrm{~b}-11$ e and Additional file 4). GO covered three parts, namely, biological process, cellular component, and molecular function terms. The biological processes significantly regulated by the 25 candidate targets of sorafenib for HCC treatment were MAPK cascade (GO: 0000165), epidermal growth factor receptor signaling pathway (GO: 0007173), negative regulation of apoptotic process (GO: 0043066), cell division (GO: 0051301), and positive regulation of fibroblast proliferation (GO: 0048146) (Figure 11b). The 25 candidate targets and their 50 frequently associated genes were primarily located in the cellular components cytosol (GO: 0005829), nucleus (GO: 0005634), nucleoplasm (GO: 0005654), membrane raft (GO: 0045121), and cyclin-dependent protein kinase holoenzyme complex (GO: 0000307). The candidate targets exerted their molecular functions through Ras guanyl-nucleotide exchange factor activity (GO: 0005088), ATP binding (GO: 0005524), protein binding (GO: 0005515), protein kinase activity (GO: 0004672), and kinase activity (GO: 0016301). Most notably, the primary pathways affected by the candidate targets were hepatitis B (hsa05161), cell cycle (hsa04110), pathways in cancer (hsa05200), FoxO signaling pathway (hsa04068), and ErbB signaling pathway (hsa04012).

\section{Discussion}

Accumulating evidence shows that CYPs are concerned not only with biotransformation of carcinogens and antineoplastic drugs but also serve as vital therapeutic targets of HCC [32]. Previous studies suggested that abnormal expression levels of several CYPs are involved in individualized treatment and prognosis of patients with HCC $[4,7]$. However, the exact roles of key CYP members in HCC must be 
clarified. In the present study, the expression and prognosis of 12 CYPs of HCC targets from the perspective of network pharmacology were comprehensively analyzed.

$\mathrm{HCC}$ is a disease predominant among men that is related to estrogen metabolism. CYP1A2 can metabolize 17beta-estradiol to generate the potent antitumor metabolite 2-methoxyestradiol. The expression level of CYP1A2 remarkably affects HCC development and progression and contributes to the gender disparity of HCC [33]. The expression level of CYP1A2 is markedly decreased in HCC [6]. Hence, the determination of CYP protein expression profile is useful for personalized HCC treatment [4]. The results of the present study showed that the expression level of CYP1A2 dramatically decreased in HCC, and its low expression level was correlated with individual cancer stages and tumor grades. However, a low expression level of CYP1A2 was not significantly correlated with the OS of patients with HCC, indicating that this enzyme may not be a potential prognostic marker in patients with HCC.

CYP27A1 is a unique enzyme that converts cholesterol to 27-hydroxycholesterol and bile acids to regulate cholesterol homeostasis. CYP27A1 is reportedly associated with prostate cancer pathogenesis [34] and a potential target of breast cancer [35]. The expression level of CYP27A1 is reduced in hepatitis $C$ virus (HCV)-associated HCC, and thus it may serve as a marker of treatment target for HCV-associated HCC [36]. In the present study, the expression level of CYP27A1 remarkably decreased in different stages and tumor grades of HCC and was correlated with the OS of patients with HCC. In addition, the mRNA expression level of CYP27A1 was significantly correlated with the OS of patients with HCC patients who received sorafenib, suggesting that this enzyme can be developed into a potential predictor biomarker for the prognosis of patients with HCC who receive sorafenib.

CYP2A6 and CYP 2A7 are key drug metabolic enzymes that mainly localize to the endoplasmic reticulum of the liver. CYP2A6 exerts a key effect on the outcome of resected gastric cancer [37] and HCC [38] via 5fluorouracil metabolism. As the metabolic-activating enzyme of most tobacco carcinogens, CYP2A6 is notably involved with the risk of lung cancer [39] and is a crucial clinical consideration for personalized medicine [40]. The expression levels of CYP2A6 and CYP2A7 are lower in patients with hepatitis B virus and HCV than those in healthy people [41]. Moreover, patients with HCC with downregulated CYP2A6 exhibit the worse OS [42]. In the present study, the expression levels of CYP2A6 and CYP2A7 were low and negatively correlated with tumor stages and grades. By contrast, the mRNA expression levels of CYP2A6 and CYP2A7 were high and significantly correlated with favorable OS in all patients with HCC. CYP2A7 was also significantly correlated with the OS of patients HCC who received sorafenib therapy.

CYP2B6 is related to testosterone metabolism; a decline in CYP2B6 activity contributes to an increased risk of breast cancer [43]. CYP2B6 also plays a crucial part in the progression of prostate cancer and may serve as a prognostic predictor for this malignancy [44]. CYP2C8 and CYP2C9 are downregulated in patients with HCC and regarded as candidate prognostic biomarker for this disease [45] and for patients with HCC following hepatectomy [46]. The proliferation of esophageal cancer is efficiently inhibited in patients with highly expressed CYP2C9 [47]. Moreover, CYP2C9 is a hub gene that affects the transformation from hepatic cirrhosis into $\operatorname{HCC}[48,49]$. In the present work, the mRNA expression levels 
of CYP2B6, CYP2C8, and CYP2C9 remarkably decreased and were negatively correlated with individual cancer stages and tumor grades. Overexpression of CYP2C8 and CYP2C9 was significantly correlated with good prognosis in 364 patients with HCC. Interestingly, no significant correlation was found between CYP2B6 overexpression and the OS of the 364 patients. Nevertheless, high CYP2B6 expression was remarkably correlated with favorable OS in 29 patients with HCC who received sorafenib.

CYP2E1 is mainly distributed in the endoplasmic reticulum and mitochondria of the liver. This enzyme mediates the metabolic activation of diethylnitrosamine, which is linked to hepatocarcinogenesis. A high CYP2E1 expression and activity may be a risk factor for HCC induced by diethylnitrosamine [50]. Downregulated CYP2E1 is regarded as a candidate prognostic biomarker in HCC [51]. Few reports are available concerning CYP2J2 expression in patients with HCC; CYP2J2 overexpression remarkably promotes the proliferation of HepG2 cells and affects the resistance to antitumor agents [52]. In the present study, the expression levels of CYP2E1 and CYP2J2 were low in patients with HCC, and their mRNA expression levels were negatively correlated with tumor grades and stages. High CYP2E1 expression was strongly correlated with favorable prognosis in patients with HCC. Moreover, no significant correlation was observed between CYP2J2 expression and OS in patients with HCC.

CYP3A4 is concerned with the metabolism of probably half of all drugs in clinical use. Sorafenib is chiefly metabolized by CYP3A4, and abnormal CYP3A4 expression will affect the exposure of sorafenib, resulting in different therapeutic effects. A decrease in CYP3A4 expression is considered an independent predictor for early recurrence of HCC [53]. Given that HCC is more prevalent in males than in females, low CYP3A4 expression in males may contribute to gender differences in morbidity [54]. Moreover, CYP3A5 alone [55] or combined with CYP3A4 are candidate prognostic markers for patients with HCC [9]. CYP3A5 may interact with CYP3A4 and affect the transcription and activity of CYP3A4 [56]. Additionally, CYP4A11 expression is reduced by $85 \%$ [6] and is recognized as a candidate diagnostic and prognostic biomarker for patients with HCC [10]. In the present study, the mRNA expression levels of CYP3A4 and CYP4A11 markedly decreased in patients with $\mathrm{HCC}$ and were negatively correlated with tumor stages and grades. The fold change of CYP3A5 expression ranged from -1.809 to -2.771 in the ONCOMINE database, indicating that CYP3A5 expression was low in patients with HCC. However, no significant difference in CYP3A5 expression was found between normal liver and HCC tissues. Additionally, high expression levels of CYP3A4, CYP3A5 and CYP4A11 were significantly correlated with good prognosis of with HCC. CYP3A5 overexpression was strongly correlated with patients with HCC who received sorafenib therapy, indicating that this enzyme may be a potential prognostic predictor for patients with $\mathrm{HCC}$ receiving sorafenib.

The effects of genetic alterations in the 12 CYPs and their relationship with the prognosis of patients with HCC were investigated. The rate of genetic alteration in individual CYP members varied from $5 \%$ to $9 \%$. The genetic alterations did not markedly affect the prognosis of patients with HCC. Moreover, the functions and pathways of the 12 CYPs and their 50 frequently altered neighboring genes in patients with HCC were analyzed. The genes were principally enriched in the chemical carcinogenesis (hsa05204) and drug metabolism - cytochrome P450 (hsa00982) pathways.

Page 13/31 
Sorafenib is the only first-line agent available for $\mathrm{HCC}$ treatment. The present results suggested that high expression levels of CYP27A1, CYP2A7, CYP2B6, CYP2C9, and CYP3A5 were considerably correlated with favorable OS in 29 patients who received sorafenib therapy. These findings may provide new insights into individualized HCC treatment via sorafenib administration. To clarify further the pharmacological mechanism of sorafenib against HCC, we conducted a network pharmacology analysis. Among the targets of sorafenib for HCC treatment, CYP1A2, CYP2B6, CYP2C8, CYP2C9, CYP3A4, and CYP3A5 were recognized as a subgroup of the PPI network. Hence, CYP2B6, CYP2C9, and CYP3A5 may be therapeutic targets and prognostic biomarkers of sorafenib for HCC treatment. Moreover, the candidate targets and their 50 closely related genes were enriched in the pathways hepatitis $B$, cell cycle, pathways in cancer, FoxO signaling pathway, and ErbB signaling pathway. The FoxO $[57,58]$ and ErbB signaling pathways [59] are involved in the proliferation and apoptosis of HCC cells, and they could affect the chemotherapeutic effects of sorafenib against HCC $[60,61]$. Sorafenib may exert its antihepatocarcinoma effects by regulating the FoxO and ErbB signaling pathways. The network pharmacology analysis conducted herein was greatly affected by the construction and improvement of the databases. Thus, the present findings require further validation.

\section{Conclusion}

Our findings suggested that the mRNA expression levels of the 12 CYPs (CYP1A2, 27A1, 2A6, 2A7, 2B6, $2 \mathrm{C} 8,2 \mathrm{C} 9,2 \mathrm{E} 1,2 \mathrm{~J} 2,3 \mathrm{~A} 4,3 \mathrm{~A} 5$, and 4A11) remarkably decreased in patients with HCC. Except for CYP3A5, these enzymes were significantly negatively correlated with tumor stages and grades. High expression levels of nine CYPs, namely, CYP27A1, 2A6, 2A7, 2C8, 2C9, 2E1, 3A4, 3A5, and 4A11, were strongly correlated with good prognosis of patients with HCC. These results indicated that these 9 CYPs may function as candidate prognostic predictors for HCC. Moreover, overexpression of CYP27A1, CYP2A7, CYP2B6, CYP2C9, and CYP3A5 were highly correlated with favorable OS of patient with HCC who received sorafenib therapy. The results of network pharmacology analysis of sorafenib for HCC treatment indicated that this drug may exert its antihepatocarcinoma action by regulating the FoxO and ErbB signaling pathways. Characterization of the expression and prognosis of the 12 CYPs in HCC from the perspective of network pharmacology offers novel insights into the discovery of therapeutic targets and prognostic markers for patients with HCC.

\section{Declarations}

\section{Acknowledgements}

None

\section{Author's contributions}

Conceptualization, P.J. and Y.S.; Data curation, Q.W.; Funding acquisition, P.J. and Y.W.; Investigation, P.J. and Q.W.; Software, P.J. and Q.W.; Supervision, K.L.; Validation, Y.W.; Visualization, K.L.; Writing - original 
draft, P.J. and Q.W.; Writing - review \& editing, Y.S. All authors read and approved the submitted version.

\section{Funding}

This work was funded by the National Natural Science Foundation of China [No. 81803832] and the Fundamental Research Funds for the Central Universities [WK9110000113].

\section{Availability of data and materials}

The data generated during and/or analysed during the current study are available from the corresponding author on reasonable request.

\section{Ethics approval and consent to participate}

Not applicable.

\section{Consent for publication}

Not applicable

\section{Competing interests}

No potential conflict of interest was reported by the authors.

\section{Author details}

${ }^{1}$ Department of Pharmacy, Division of Life Sciences and Medicine, The First Affiliated Hospital of USTC, University of Science and Technology of China, Hefei, Anhui, 230031, China.

2 Department of Pharmacy, Anhui University of Chinese Medicine, Hefei, Anhui, 230012, China.

${ }^{3}$ Institute of Pharmaceutics, Anhui Academy of Chinese Medicine, Hefei, Anhui, 230012, China.

${ }^{4}$ Anhui Province Key Laboratory of Pharmaceutical Formulation Technology and Application (Anhui University of Chinese Medicine), Hefei, Anhui, 230012, China.

\section{References}

1. Siegel RL, Miller KD, Jemal A. Cancer statistics, 2019. CA Cancer J Clin. 2019;69:7-34.

2. Li Y, Gao ZH, Qu XJ. The adverse effects of sorafenib in patients with advanced cancers. Basic Clin Pharmacol Toxicol. 2015;116:216-21.

3. Zhai S, Huang Q, Liao X, Yin S. Study on the Drug Targets and Molecular Mechanisms of Rhizoma Curcumae in the Treatment of Nasopharyngeal Carcinoma Based on Network Pharmacology. Evid Based Complement Alternat Med. 2020;2020:2606402. 
4. Yan T, Lu L, Xie C, Chen J, Peng X, Zhu L, Wang Y, Li Q, Shi J, Zhou F, et al. Severely Impaired and Dysregulated Cytochrome P450 Expression and Activities in Hepatocellular Carcinoma: Implications for Personalized Treatment in Patients. Mol Cancer Ther. 2015;14:2874-86.

5. Yan T, Gao S, Peng X, Shi J, Xie C, Li Q, Lu L, Wang Y, Zhou F, Liu Z, Hu M. Significantly decreased and more variable expression of major CYPs and UGTs in liver microsomes prepared from HBVpositive human hepatocellular carcinoma and matched pericarcinomatous tissues determined using an isotope label-free UPLC-MS/MS method. Pharm Res. 2015;32:1141-57.

6. Chen H, Shen ZY, Xu W, Fan TY, Li J, Lu YF, Cheng ML, Liu J. Expression of P450 and nuclear receptors in normal and end-stage Chinese livers. World J Gastroenterol. 2014;20:8681-90.

7. Liu ZZ, Yan LN, Dong CN, Ma N, Yuan MN, Zhou J, Gao P. Cytochrome P450 family members are associated with fast-growing hepatocellular carcinoma and patient survival: An integrated analysis of gene expression profiles. Saudi J Gastroenterol. 2019;25:167-75.

8. Shuaichen L, Guangyi W. Bioinformatic analysis reveals CYP2C9 as a potential prognostic marker for HCC and liver cancer cell lines suitable for its mechanism study. Cell Mol Biol (Noisy-le-grand). 2018;64:70-4.

9. Yu T, Wang X, Zhu G, Han C, Su H, Liao X, Yang C, Qin W, Huang K, Peng T. The prognostic value of differentially expressed CYP3A subfamily members for hepatocellular carcinoma. Cancer Manag Res. 2018;10:1713-26.

10. Eun HS, Cho SY, Lee BS, Kim S, Song IS, Chun K, Oh CH, Yeo MK, Kim SH, Kim KH. Cytochrome P450 4A11 expression in tumor cells: A favorable prognostic factor for hepatocellular carcinoma patients. J Gastroenterol Hepatol. 2019;34:224-33.

11. Hu DG, Marri S, McKinnon RA, Mackenzie PI, Meech R. Deregulation of the Genes that Are Involved in Drug Absorption, Distribution, Metabolism, and Excretion in Hepatocellular Carcinoma. J Pharmacol Exp Ther. 2019;368:363-81.

12. Rhodes DR, Yu J, Shanker K, Deshpande N, Varambally R, Ghosh D, Barrette T, Pandey A, Chinnaiyan AM. ONCOMINE: a cancer microarray database and integrated data-mining platform. Neoplasia. 2004;6:1-6.

13. Ning G, Huang YL, Zhen LM, Xu WX, Jiao Q, Yang FJ, Wu LN, Zheng YY, Song J, Wang YS, et al. Transcriptional expressions of Chromobox $1 / 2 / 3 / 6 / 8$ as independent indicators for survivals in hepatocellular carcinoma patients. Aging (Albany NY). 2018;10:3450-73.

14. Chandrashekar DS, Bashel B, Balasubramanya SAH, Creighton CJ, Ponce-Rodriguez I, Chakravarthi B, Varambally S. UALCAN: A Portal for Facilitating Tumor Subgroup Gene Expression and Survival Analyses. Neoplasia. 2017;19:649-58.

15. Thul PJ, Lindskog C. The human protein atlas: A spatial map of the human proteome. Protein Sci. 2018;27:233-44.

16. Menyhart O, Nagy A, Gyorffy B. Determining consistent prognostic biomarkers of overall survival and vascular invasion in hepatocellular carcinoma. R Soc Open Sci. 2018;5:181006. 
17. Tomczak K, Czerwinska P, Wiznerowicz M. The Cancer Genome Atlas (TCGA): an immeasurable source of knowledge. Contemp Oncol (Pozn). 2015;19:A68-77.

18. Gao J, Aksoy BA, Dogrusoz U, Dresdner G, Gross B, Sumer SO, Sun Y, Jacobsen A, Sinha R, Larsson E, et al. Integrative analysis of complex cancer genomics and clinical profiles using the cBioPortal. Sci Signal. 2013;6:pl1.

19. Cerami E, Gao J, Dogrusoz U, Gross BE, Sumer SO, Aksoy BA, Jacobsen A, Byrne CJ, Heuer ML, Larsson $\mathrm{E}$, et al. The cBio cancer genomics portal: an open platform for exploring multidimensional cancer genomics data. Cancer Discov. 2012;2:401-4.

20. Szklarczyk D, Morris JH, Cook H, Kuhn M, Wyder S, Simonovic M, Santos A, Doncheva NT, Roth A, Bork P, et al. The STRING database in 2017: quality-controlled protein-protein association networks, made broadly accessible. Nucleic Acids Res. 2017;45:D362-D8.

21. Huang da W, Sherman BT, Lempicki RA. Systematic and integrative analysis of large gene lists using DAVID bioinformatics resources. Nat Protoc. 2009;4:44-57.

22. Wang Y, Zhang S, Li F, Zhou Y, Zhang Y, Wang Z, Zhang R, Zhu J, Ren Y, Tan Y, et al. Therapeutic target database 2020: enriched resource for facilitating research and early development of targeted therapeutics. Nucleic Acids Res. 2020;48:D1031-D41.

23. Wishart DS, Feunang YD, Guo AC, Lo EJ, Marcu A, Grant JR, Sajed T, Johnson D, Li C, Sayeeda Z, et al. DrugBank 5.0: a major update to the DrugBank database for 2018. Nucleic Acids Res. 2018;46:D1074-D82.

24. Daina A, Michielin O, Zoete V. SwissTargetPrediction: updated data and new features for efficient prediction of protein targets of small molecules. Nucleic Acids Res. 2019;47:W357-W64.

25. Su WH, Chao CC, Yeh SH, Chen DS, Chen PJ, Jou YS. OncoDB.HCC: an integrated oncogenomic database of hepatocellular carcinoma revealed aberrant cancer target genes and loci. Nucleic Acids Res. 2007;35:D727-31.

26. Lee L, Wang K, Li G, Xie Z, Wang Y, Xu J, Sun S, Pocalyko D, Bhak J, Kim C, et al. Liverome: a curated database of liver cancer-related gene signatures with self-contained context information. BMC Genomics. 2011;12 Suppl 3:S3.

27. Roessler S, Jia HL, Budhu A, Forgues M, Ye QH, Lee JS, Thorgeirsson SS, Sun Z, Tang ZY, Qin LX, Wang XW. A unique metastasis gene signature enables prediction of tumor relapse in early-stage hepatocellular carcinoma patients. Cancer Res. 2010;70:10202-12.

28. Wurmbach E, Chen YB, Khitrov G, Zhang W, Roayaie S, Schwartz M, Fiel I, Thung S, Mazzaferro V, Bruix J, et al. Genome-wide molecular profiles of HCV-induced dysplasia and hepatocellular carcinoma. Hepatology. 2007;45:938-47.

29. Chen X, Cheung ST, So S, Fan ST, Barry C, Higgins J, Lai KM, Ji J, Dudoit S, Ng IO, et al. Gene expression patterns in human liver cancers. Mol Biol Cell. 2002;13:1929-39.

30. Mas VR, Maluf DG, Archer KJ, Yanek K, Kong X, Kulik L, Freise CE, Olthoff KM, Ghobrial RM, Mclver P, Fisher R. Genes involved in viral carcinogenesis and tumor initiation in hepatitis $C$ virus-induced hepatocellular carcinoma. Mol Med. 2009;15:85-94. 
31. Guo W, Huang J, Wang N, Tan HY, Cheung F, Chen F, Feng Y. Integrating Network Pharmacology and Pharmacological Evaluation for Deciphering the Action Mechanism of Herbal Formula Zuojin Pill in Suppressing Hepatocellular Carcinoma. Front Pharmacol. 2019;10:1185.

32. Sheweita SA. Drug-metabolizing enzymes: mechanisms and functions. Curr Drug Metab. 2000;1:10732.

33. Ren J, Chen GG, Liu Y, Su X, Hu B, Leung BC, Wang Y, Ho RL, Yang S, Lu G, et al. Cytochrome P450 1A2 Metabolizes 17beta-Estradiol to Suppress Hepatocellular Carcinoma. PLoS One. 2016;11:e0153863.

34. Alfaqih MA, Nelson ER, Liu W, Safi R, Jasper JS, Macias E, Geradts J, Thompson JW, Dubois LG, Freeman MR, et al. CYP27A1 Loss Dysregulates Cholesterol Homeostasis in Prostate Cancer. Cancer Res. 2017;77:1662-73.

35. Mast N, Lin JB, Pikuleva IA. Marketed Drugs Can Inhibit Cytochrome P450 27A1, a Potential New Target for Breast Cancer Adjuvant Therapy. Mol Pharmacol. 2015;88:428-36.

36. Tsunedomi R, lizuka N, Hamamoto Y, Uchimura S, Miyamoto T, Tamesa T, Okada T, Takemoto N, Takashima M, Sakamoto K, et al. Patterns of expression of cytochrome P450 genes in progression of hepatitis C virus-associated hepatocellular carcinoma. Int J Oncol. 2005;27:661-7.

37. Jeong JH, Park SR, Ahn Y, Ryu MH, Ryoo BY, Kong SY, Yook JH, Yoo MW, Kim BS, Kim BS, Kang YK. Associations between CYP2A6 polymorphisms and outcomes of adjuvant S-1 chemotherapy in patients with curatively resected gastric cancer. Gastric Cancer. 2017;20:146-55.

38. Fushiya N, Takagi I, Nishino H, Akizuki S, Ohnishi A. Genetic polymorphisms of enzymes related to oral tegafur/uracil therapeutic efficacy in patients with hepatocellular carcinoma. Anticancer Drugs. 2013;24:617-22.

39. Johani FH, Majid MSA, Azme MH, Nawi AM. Cytochrome P450 2A6 whole-gene deletion (CYP2A6*4) polymorphism reduces risk of lung cancer: A meta-analysis. Tob Induc Dis. 2020;18:50.

40. Tanner JA, Tyndale RF. Variation in CYP2A6 Activity and Personalized Medicine. J Pers Med. 2017;7.

41. lizuka N, Oka M, Hamamoto Y, Mori N, Tamesa T, Tangoku A, Miyamoto T, Uchimura S, Nakayama H, Hamada K, Yamada-Okabe H. Altered Levels of Cytochrome P450 Genes in Hepatitis B or C Virusinfected Liver Identified by Oligonucleotide Microarray. Cancer Genomics Proteomics. 2004;1:53-8.

42. Ren X, Ji Y, Jiang X, Qi X. Downregulation of CYP2A6 and CYP2C8 in Tumor Tissues Is Linked to Worse Overall Survival and Recurrence-Free Survival from Hepatocellular Carcinoma. Biomed Res Int. 2018;2018:5859415.

43. Justenhoven C, Pentimalli D, Rabstein S, Harth V, Lotz A, Pesch B, Bruning T, Dork T, Schurmann P, Bogdanova N, et al. CYP2B6* 6 is associated with increased breast cancer risk. Int J Cancer. 2014;134:426-30.

44. Kumagai J, Fujimura T, Takahashi S, Urano T, Ogushi T, Horie-Inoue K, Ouchi Y, Kitamura T, Muramatsu M, Blumberg B, Inoue S. Cytochrome P450 2B6 is a growth-inhibitory and prognostic factor for prostate cancer. Prostate. 2007;67:1029-37. 
45. Wang X, Liao X, Yang C, Huang K, Yu T, Yu L, Han C, Zhu G, Zeng X, Liu Z, et al. Identification of prognostic biomarkers for patients with hepatocellular carcinoma after hepatectomy. Oncol Rep. 2019;41:1586-602.

46. Wang X, Yu T, Liao X, Yang C, Han C, Zhu G, Huang K, Yu L, Qin W, Su H, et al. The prognostic value of CYP2C subfamily genes in hepatocellular carcinoma. Cancer Med. 2018;7:966-80.

47. Schmelzle M, Dizdar L, Matthaei H, Baldus SE, Wolters J, Lindenlauf N, Bruns I, Cadeddu RP, Kropil F, Topp SA, et al. Esophageal cancer proliferation is mediated by cytochrome P450 2C9 (CYP2C9). Prostaglandins Other Lipid Mediat. 2011;94:25-33.

48. Jiang CH, Yuan X, Li JF, Xie YF, Zhang AZ, Wang XL, Yang L, Liu CX, Liang WH, Pang LJ, et al. Bioinformatics-based screening of key genes for transformation of liver cirrhosis to hepatocellular carcinoma. J Transl Med. 2020;18:40.

49. Fan W, Ye G. Microarray analysis for the identification of specific proteins and functional modules involved in the process of hepatocellular carcinoma originating from cirrhotic liver. Mol Med Rep. 2018;17:5619-26.

50. Gao J, Wang Z, Wang GJ, Zhang HX, Gao N, Wang J, Wang CE, Chang Z, Fang Y, Zhang YF, et al. Higher CYP2E1 Activity Correlates with Hepatocarcinogenesis Induced by Diethylnitrosamine. J Pharmacol Exp Ther. 2018;365:398-407.

51. Ma X, Zhou L, Zheng S. Transcriptome analysis revealed key prognostic genes and microRNAs in hepatocellular carcinoma. PeerJ. 2020;8:e8930.

52. Hwang GH, Park SM, Han HJ, Baek KM, Kim JS, Chang W, Lee HJ, Yun SP, Ryu JM, Lee MY. Role of cytochrome P450 2J2 on cell proliferation and resistance to an anticancer agent in hepatocellular carcinoma HepG2 cells. Oncol Lett. 2017;14:5484-90.

53. Ashida R, Okamura Y, Ohshima K, Kakuda Y, Uesaka K, Sugiura T, Ito T, Yamamoto Y, Sugino T, Urakami K, et al. CYP3A4 Gene Is a Novel Biomarker for Predicting a Poor Prognosis in Hepatocellular Carcinoma. Cancer Genomics Proteomics. 2017;14:445-53.

54. Wu Y, Yao N, Feng Y, Tian Z, Yang Y, Zhao Y. Identification and characterization of sexual dimorphismlinked gene expression profile in hepatocellular carcinoma. Oncol Rep. 2019;42:937-52.

55. Jiang F, Chen L, Yang YC, Wang XM, Wang RY, Li L, Wen W, Chang YX, Chen CY, Tang J, et al. CYP3A5 Functions as a Tumor Suppressor in Hepatocellular Carcinoma by Regulating mTORC2/Akt Signaling. Cancer Res. 2015;75:1470-81.

56. Kuang Z, Huang Z, Li Y, Yang G, Liu M, Yuan H. Overexpression of CYP3A5 attenuates inducibility and activity of CYP3A4 in HepG2 cells. Mol Med Rep. 2015;11:2868-74.

57. Lou K, Chen N, Li Z, Zhang B, Wang X, Chen Y, Xu H, Wang D, Wang H. MicroRNA-142-5p Overexpression Inhibits Cell Growth and Induces Apoptosis by Regulating FOXO in Hepatocellular Carcinoma Cells. Oncol Res. 2017;25:65-73.

58. Tan C, Liu S, Tan S, Zeng X, Yu H, Li A, Bei C, Qiu X. Polymorphisms in microRNA target sites of forkhead box $O$ genes are associated with hepatocellular carcinoma. PLoS One. 2015;10:e0119210. 
59. Liu Y, Calmel C, Desbois-Mouthon C, Sobczak-Thepot J, Karaiskou A, Praz F. Regulation of the EGFR/ErbB signalling by clathrin in response to various ligands in hepatocellular carcinoma cell lines. J Cell Mol Med. 2020.

60. Blivet-Van Eggelpoel MJ, Chettouh H, Fartoux L, Aoudjehane L, Barbu V, Rey C, Priam S, Housset C, Rosmorduc O, Desbois-Mouthon C. Epidermal growth factor receptor and HER-3 restrict cell response to sorafenib in hepatocellular carcinoma cells. J Hepatol. 2012;57:108-15.

61. Abd-Elbaset M, Mansour AM, Ahmed OM, Abo-Youssef AM. The potential chemotherapeutic effect of beta-ionone and/or sorafenib against hepatocellular carcinoma via its antioxidant effect, PPARgamma, FOXO-1, Ki-67, Bax, and Bcl-2 signaling pathways. Naunyn Schmiedebergs Arch Pharmacol. 2020.

\section{Figures}

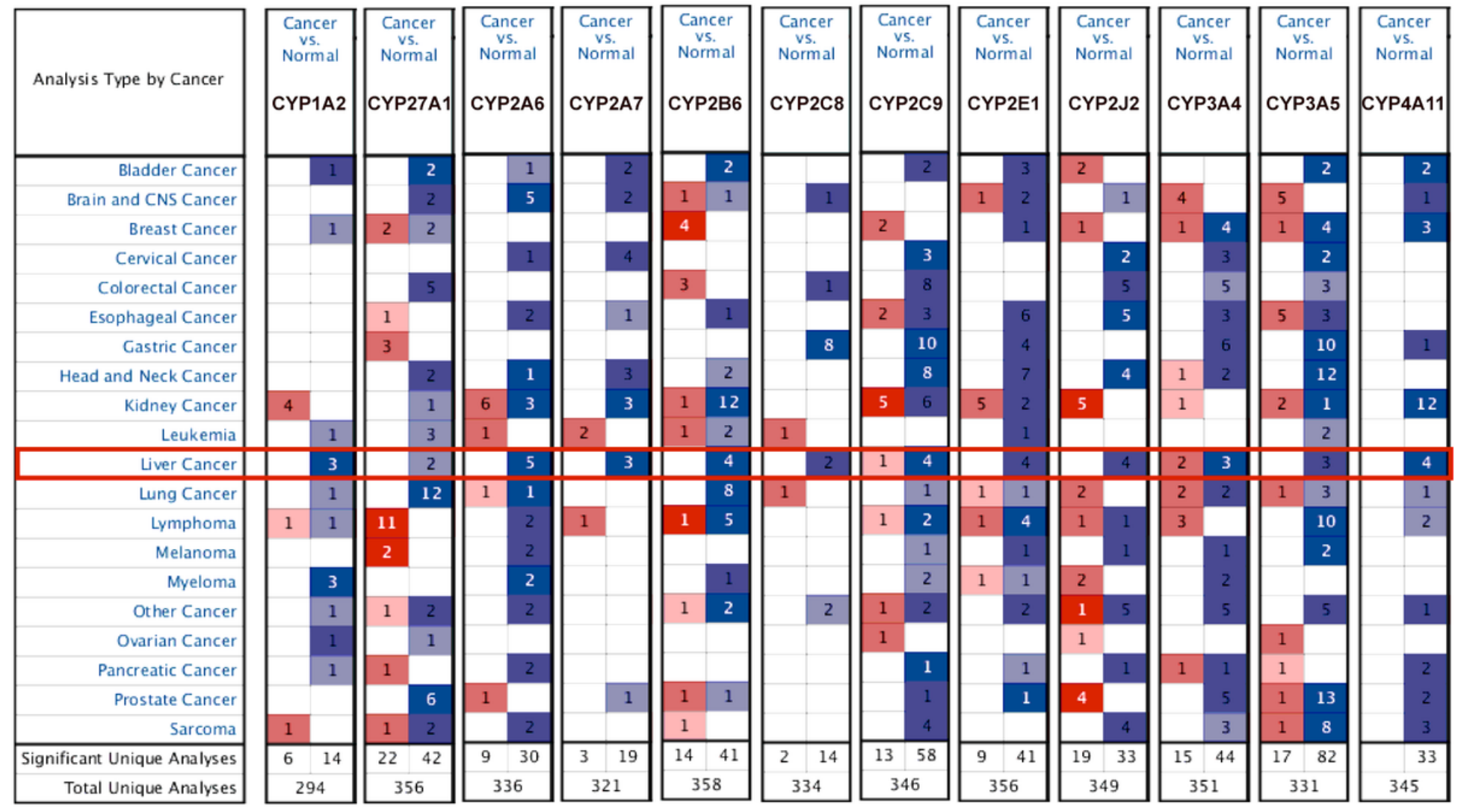

\section{Figure 1}

Transcription levels of 12 CYPs in different types of cancers (ONCOMINE). The threshold was set with the following parameters: $p$-value: 0.01 and fold change $=1.5$. 

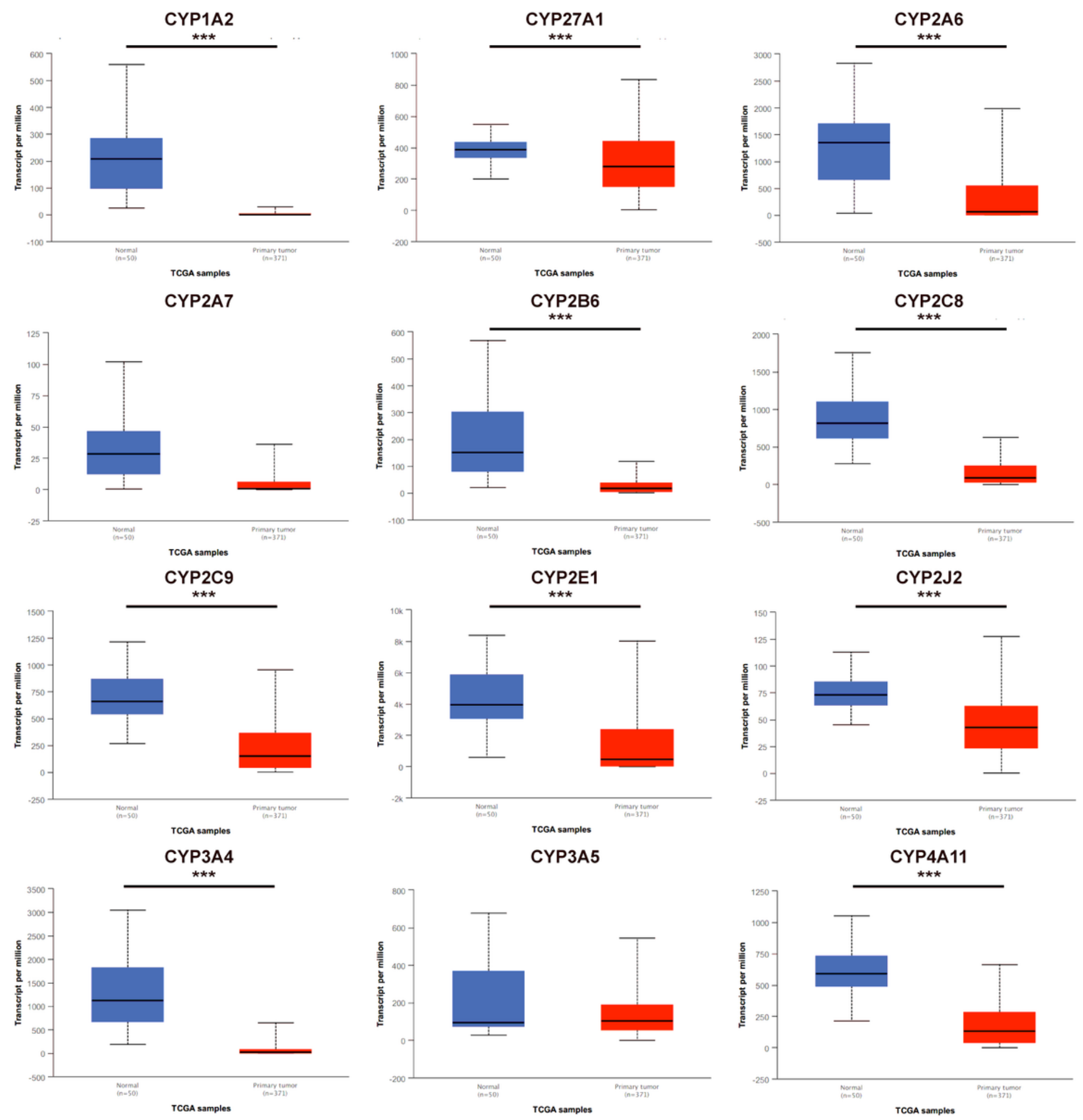

Figure 2

mRNA expression levels of 12 CYP family members in HCC tissues and normal liver tissues (UALCAN). The mRNA expression levels of 12 CYP family members (except CYP3A5) were lower in primary HCC tissues than in normal samples; $* \star \star p<0.001$. 

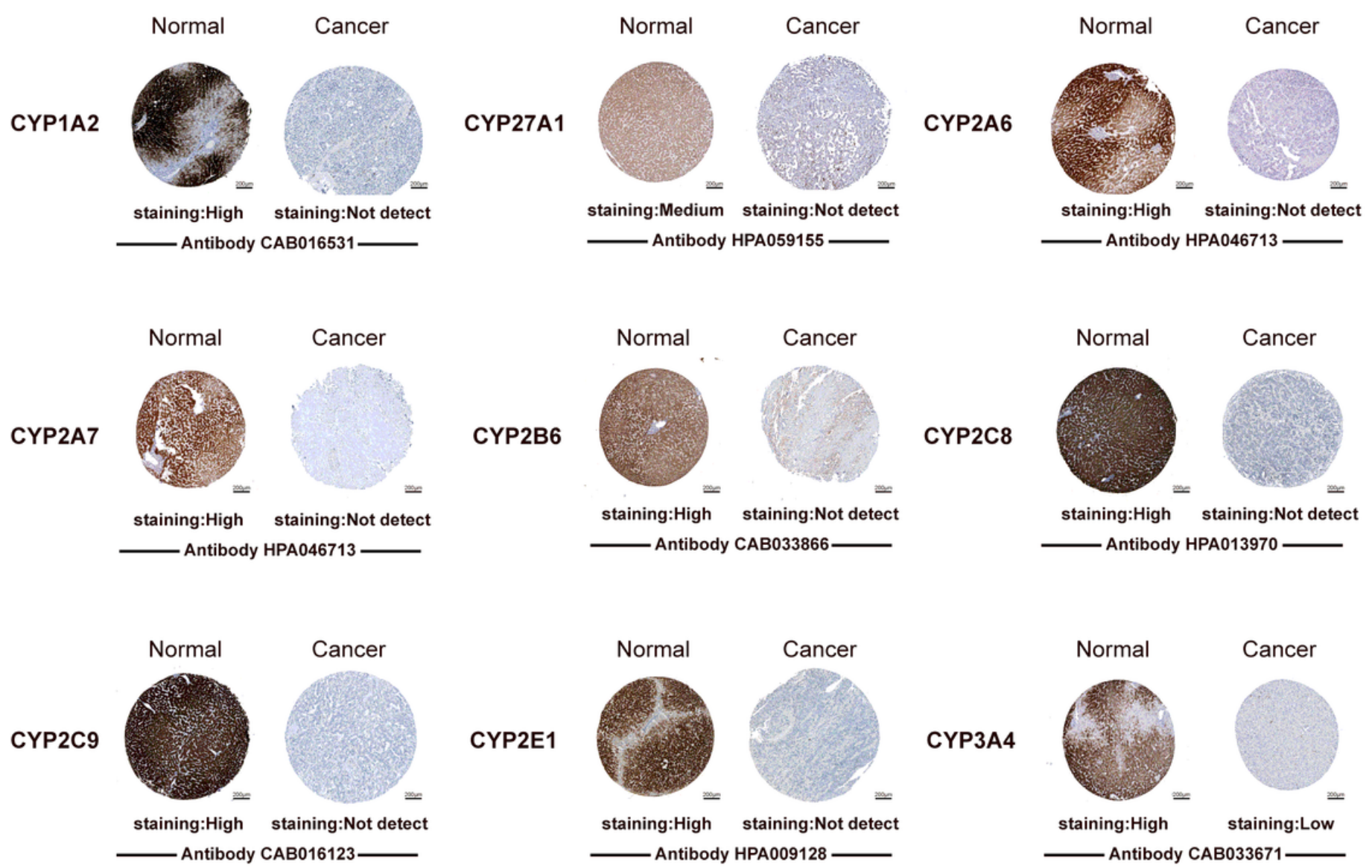

\section{Figure 3}

Representative immunohistochemistry images of the distinct CYP family members in HCC tissues and normal liver tissues (Human Protein Atlas). The immunohistochemistry images of CYP2J2, CYP3A5, and CYP4A11 were not included in Human Protein Atlas. The protein expression of nine CYPs was higher in normal liver tissues than in HCC tissues. 

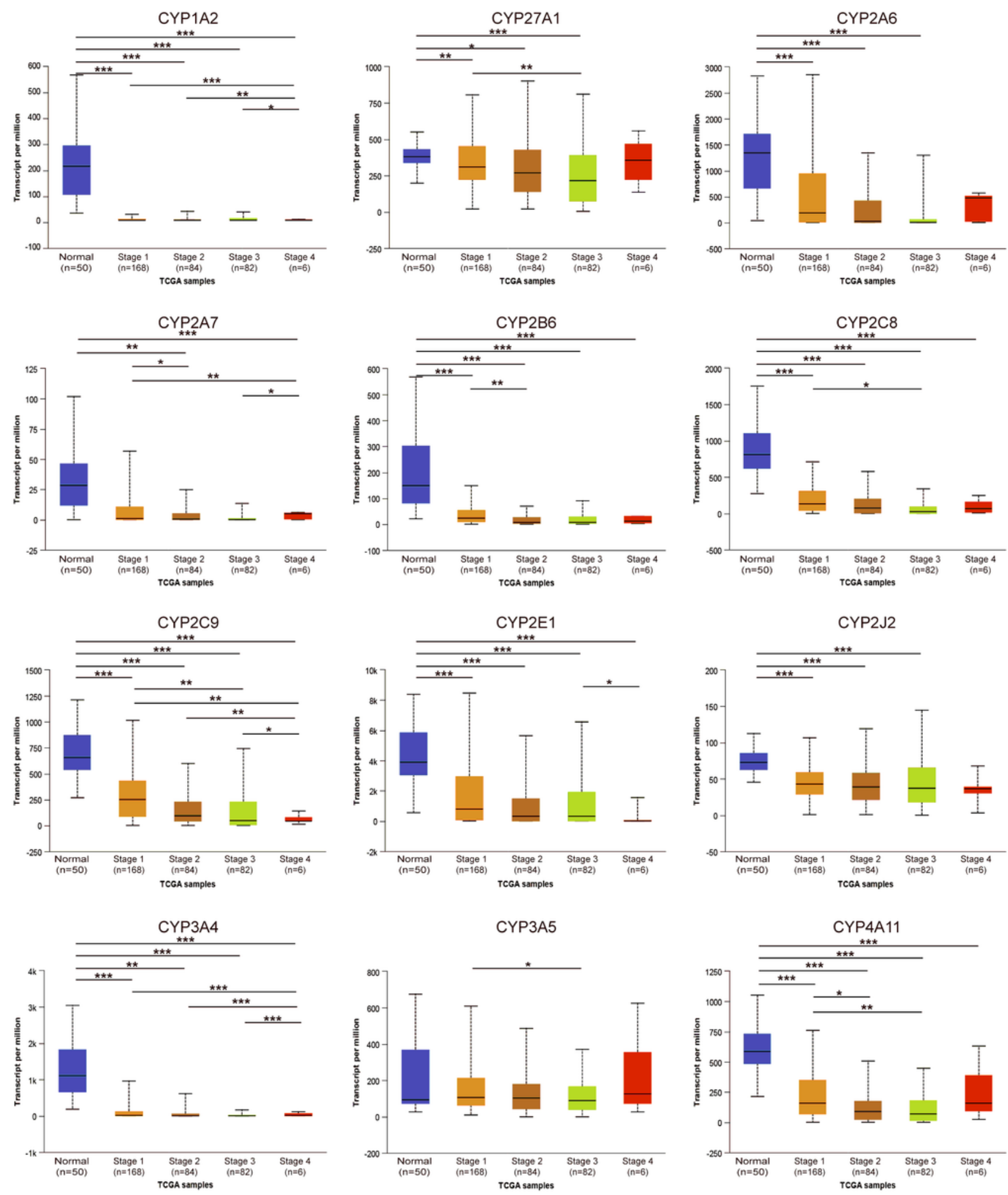

Figure 4

Relationship between the mRNA expression of 12 CYP family members and the individual cancer stages of patients with HCC. ${ }^{*} p<0.05, * * p<0.01$, and ${ }^{* * *} p<0.001$. 

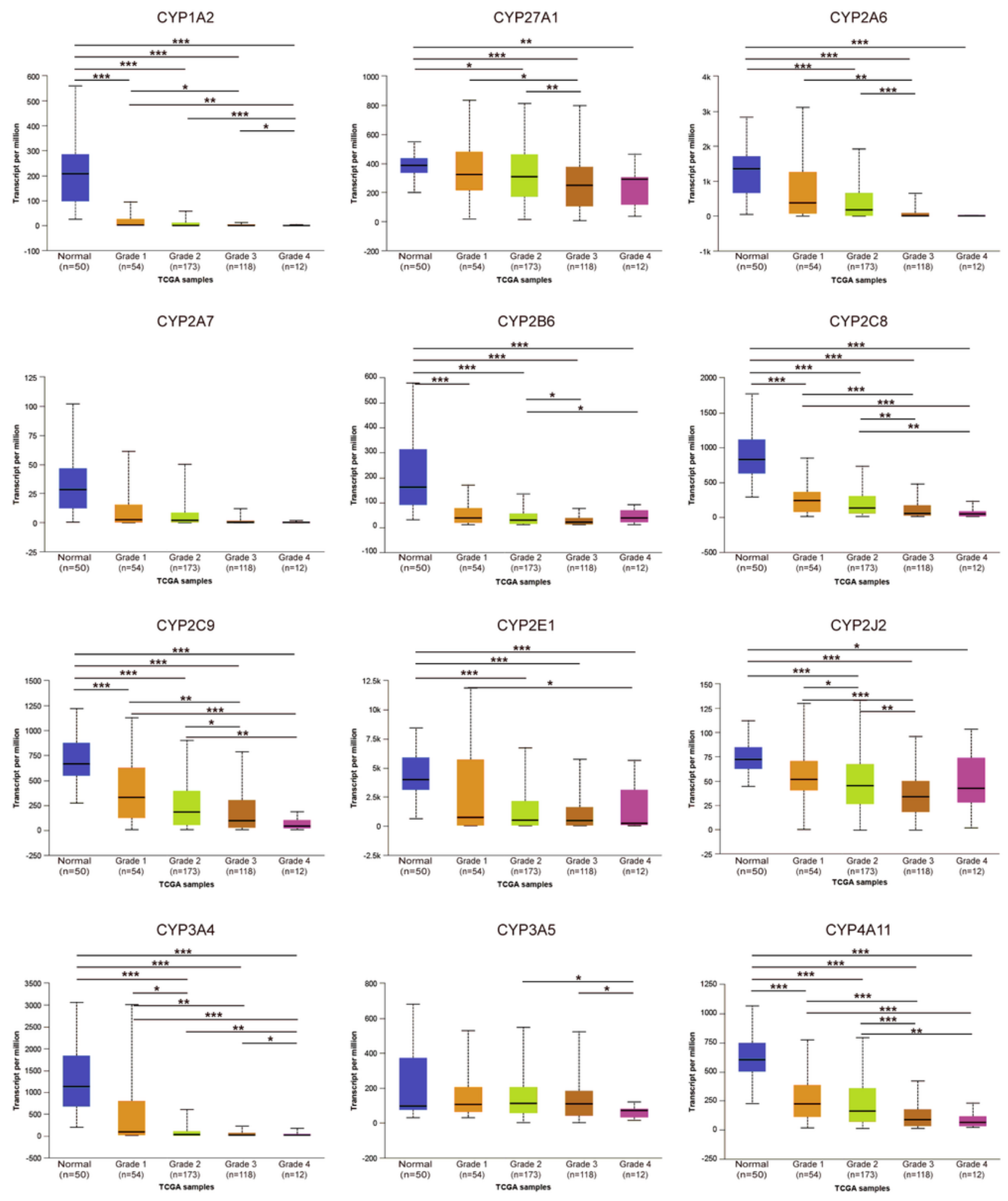

\section{Figure 5}

Relationship between the mRNA expression of 12 CYP family members and the tumor grades of patients with HCC. ${ }^{*} p<0.05, * * p<0.01$, and $* \star * p<0.001$. 

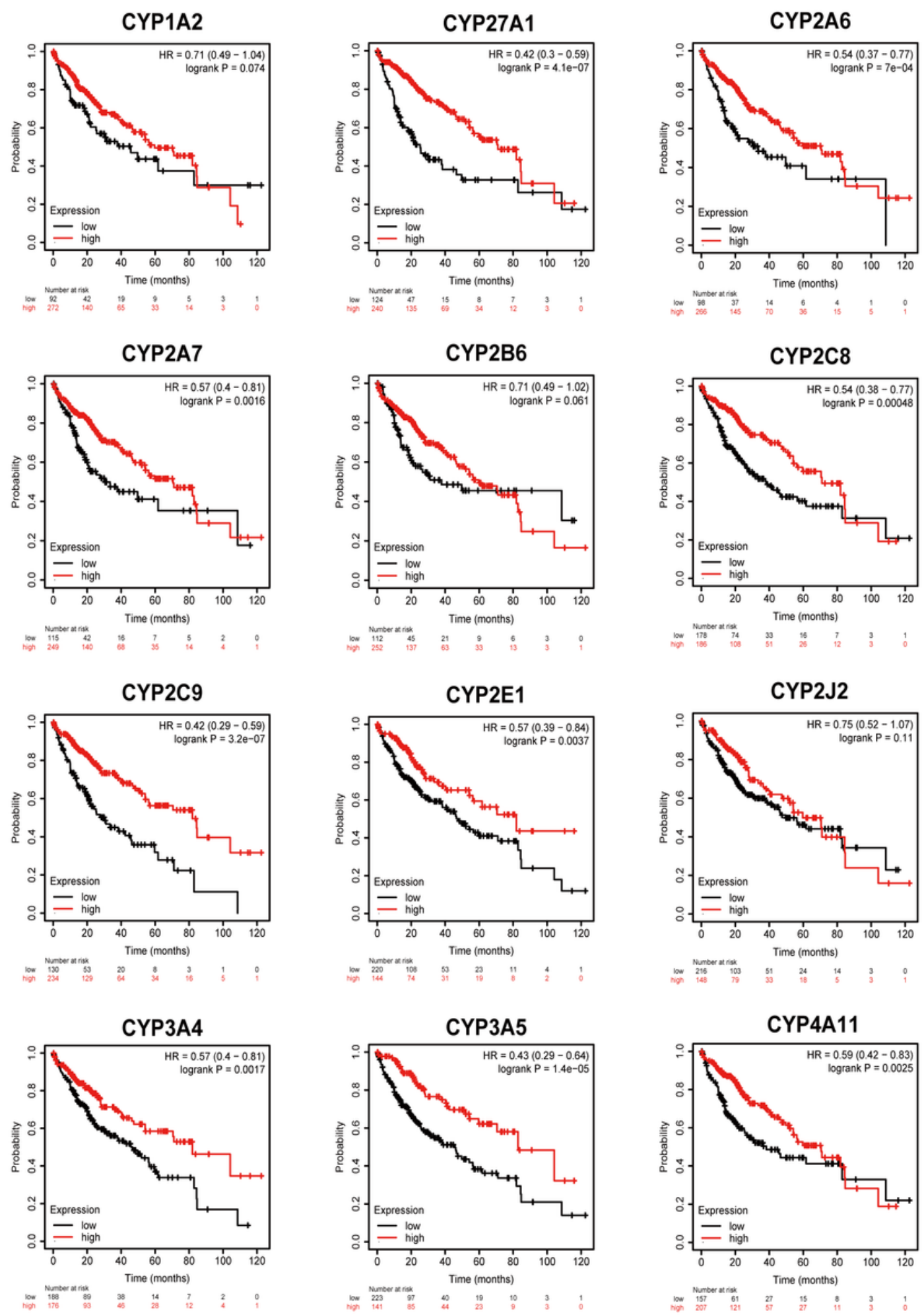

\section{Figure 6}

Prognostic value of the mRNA expression of 12 CYP family members in patients with liver cancer (Kaplan-Meier plotter). 

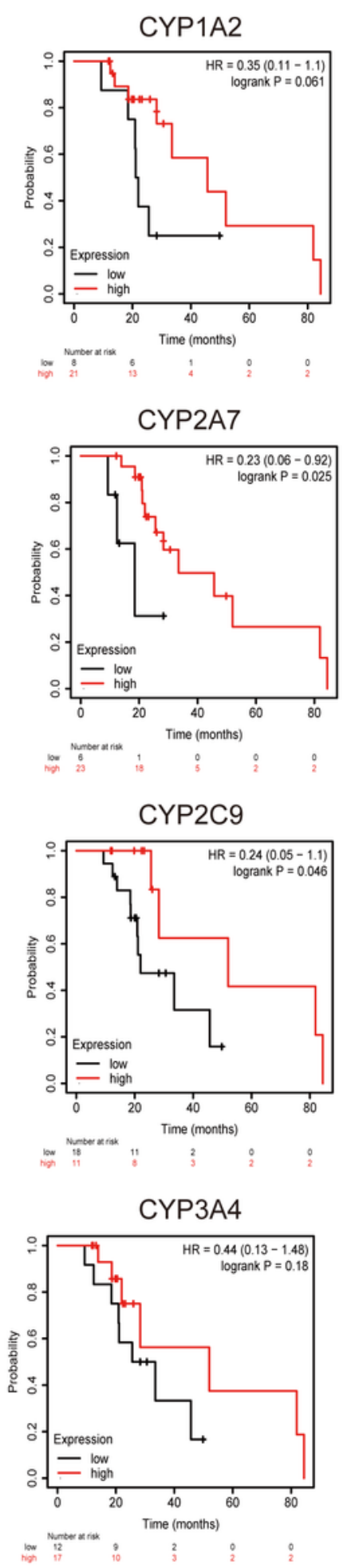
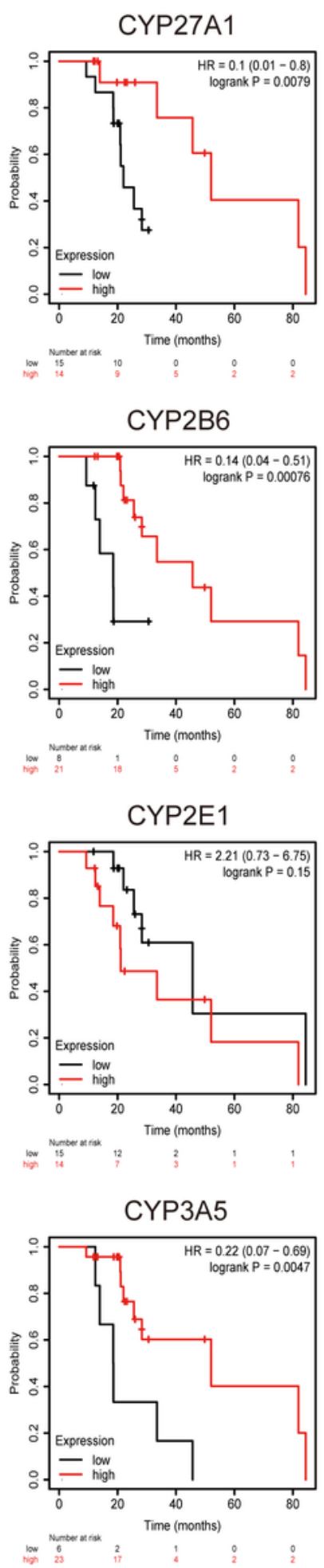
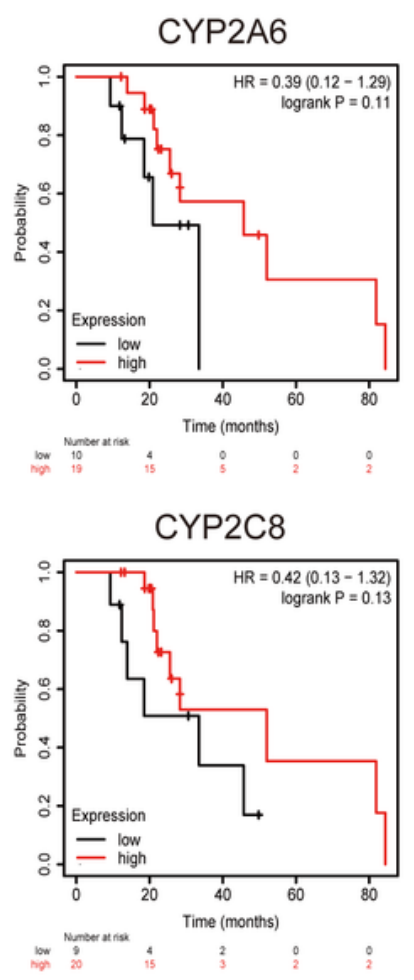

CYP2J2
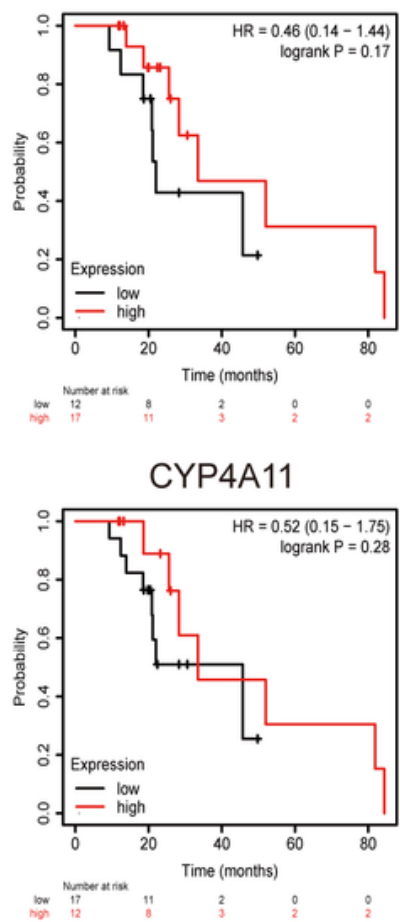

\section{Figure 7}

Prognostic value of mRNA expression of 12 CYPs family members in patients with liver cancer who received sorafenib therapy (Kaplan-Meier plotter). 
a Cancer types summary

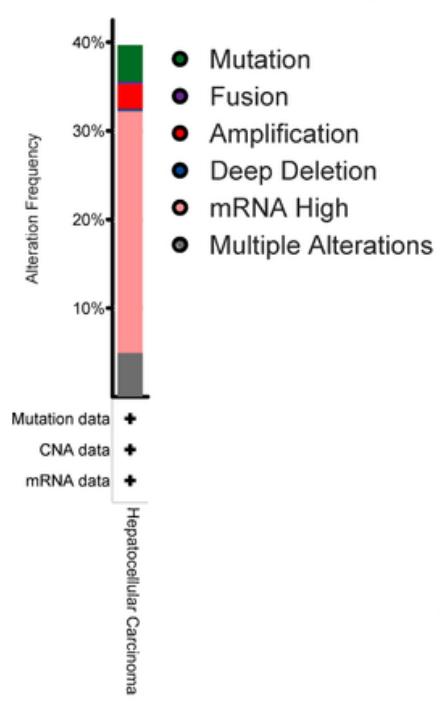

Queried genes are altered in $144(39 \%)$ of 366 queried patients/samples

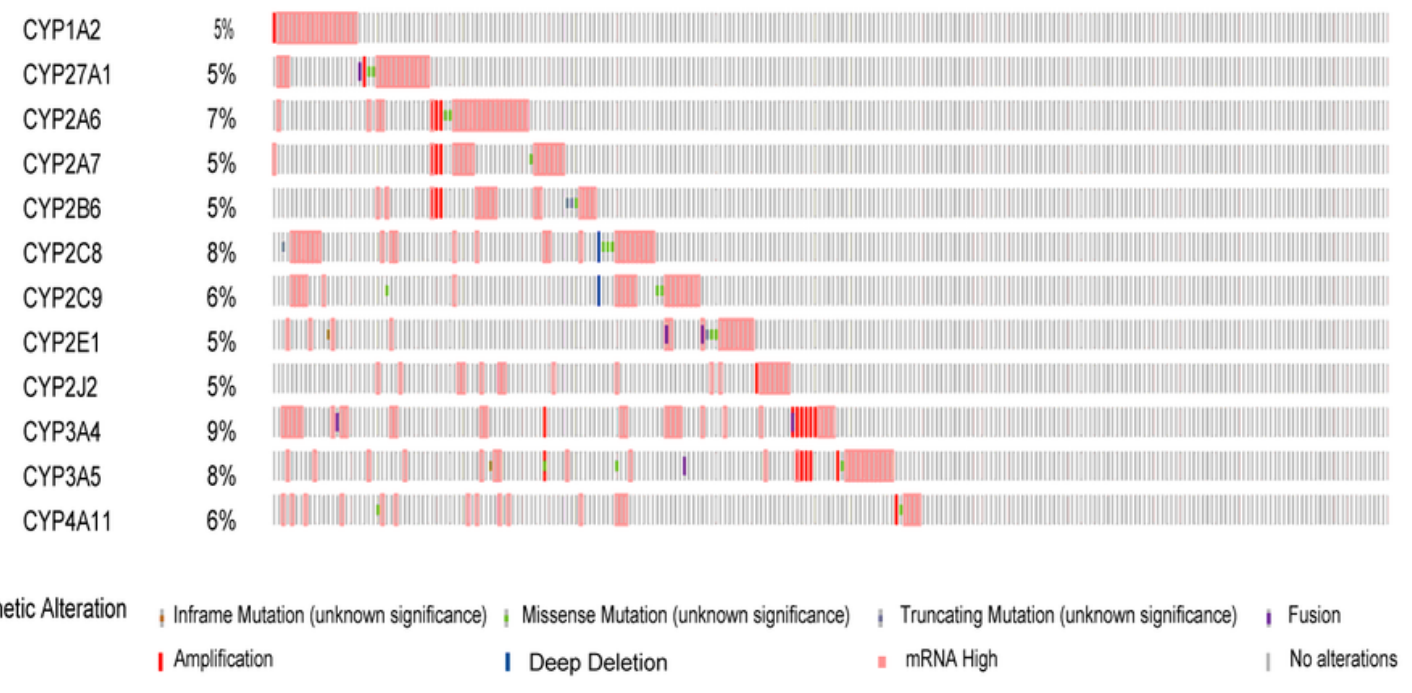

b

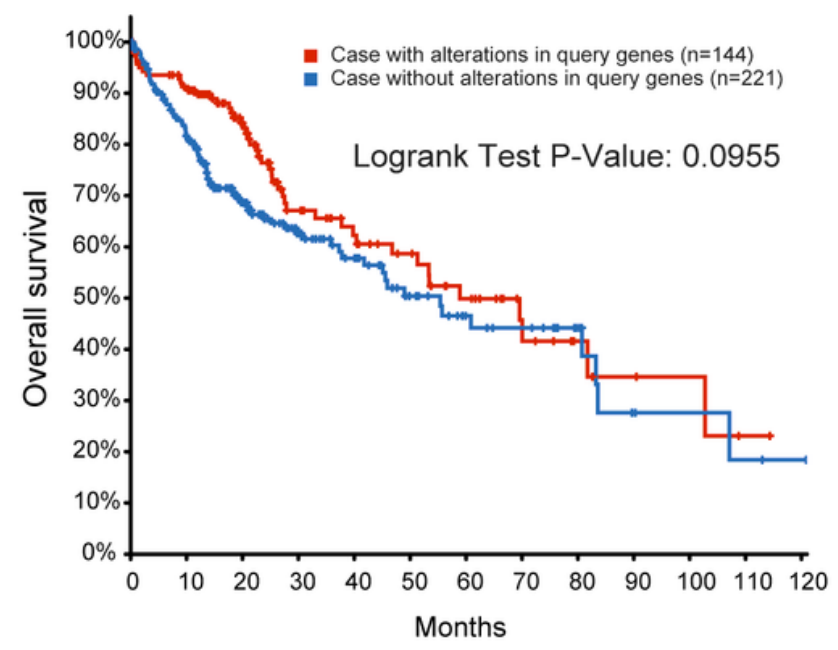

C

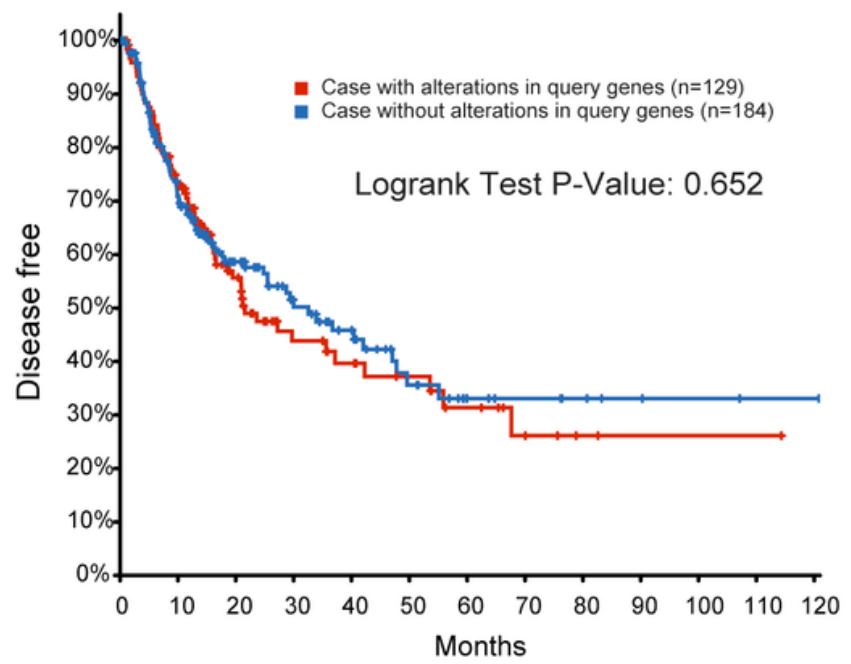

Figure 8

Genetic mutations in 12 CYPs and their association with OS and DFS of patients with HCC (cBioPortal). a The gene expression and mutation analysis of 12 CYPs in HCC (cBioPortal). b Kaplan-Meier plots comparing OS in cases with/without 12 CYP gene alterations. c Kaplan-Meier plots comparing DFS in cases with/without 12 CYP gene alterations. 


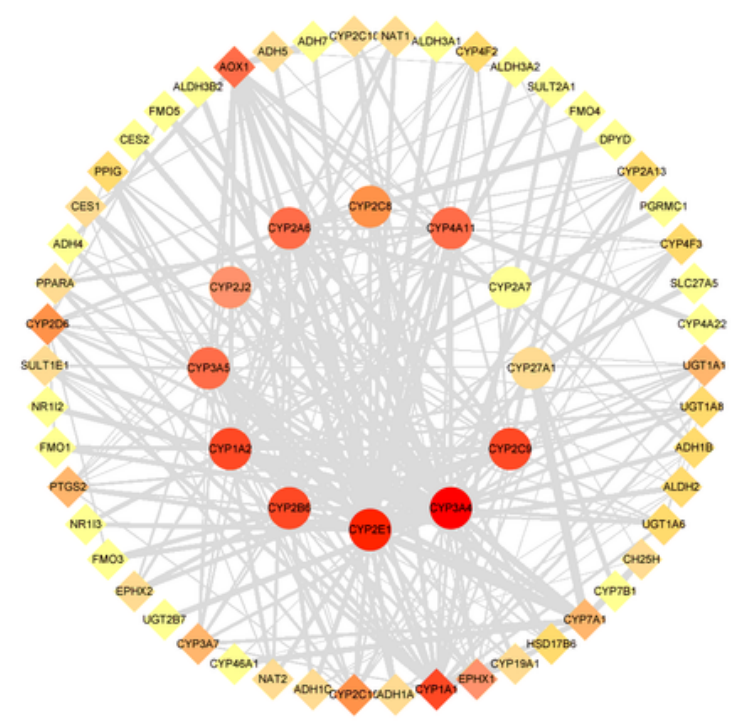

b

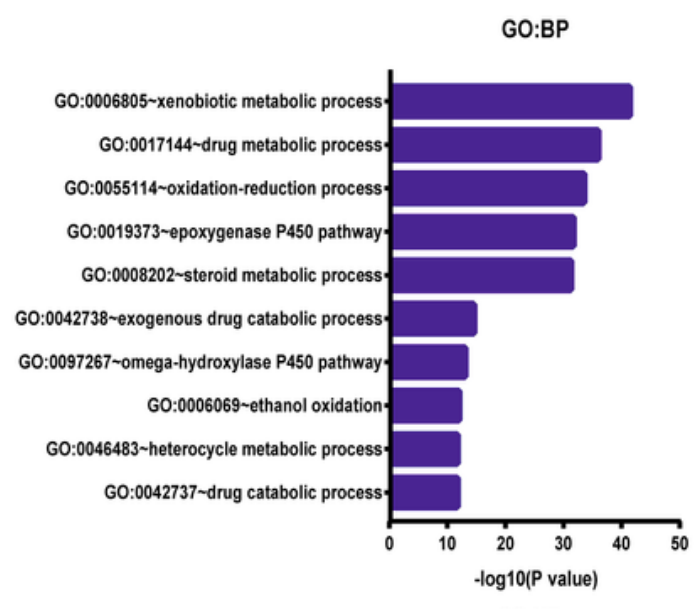

d

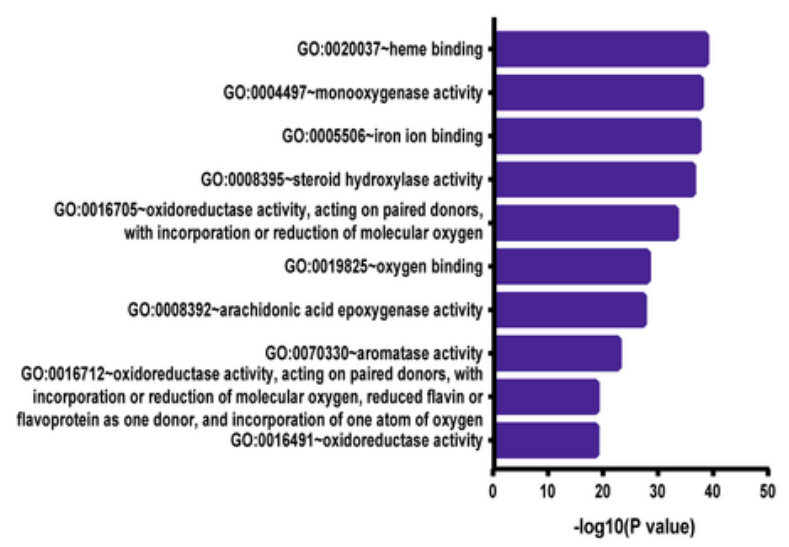

C

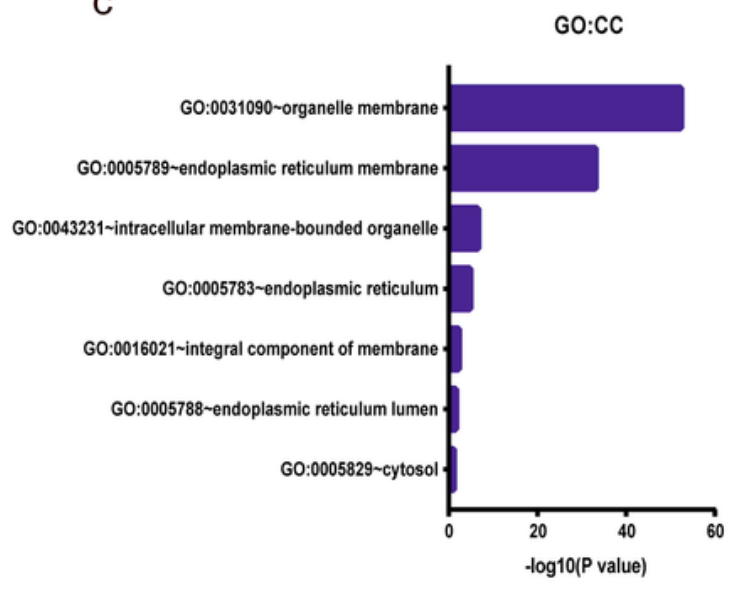

e

KEGG

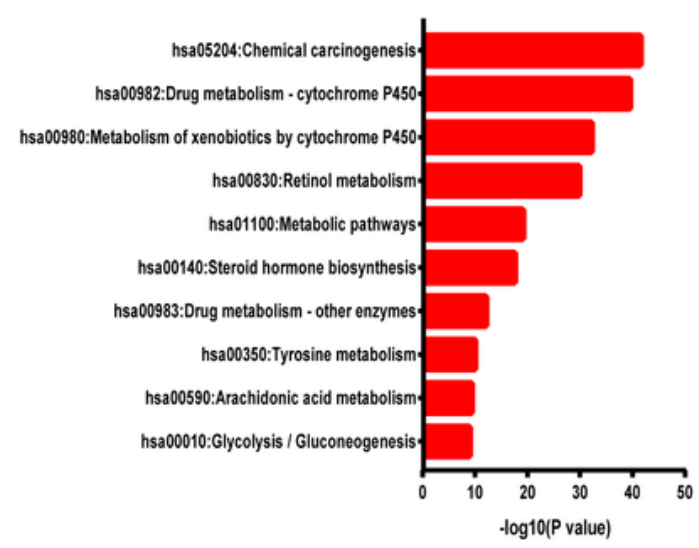

\section{Figure 9}

Function and pathway analysis of CYPs and their 50 frequently altered neighbor genes in patients with HCC (STRING and DAVID). a Protein-protein interaction (PPI) network construction by using the STING database. b-d GO functional enrichment analysis predicted the three main functions of 12 CYPs and their 50 frequently altered neighbor genes, including biological process (BP), cellular components (CC), 
and molecular functions (MF). e KEGG pathway analysis of 12 CYPs and their 50 frequently altered neighbor genes.

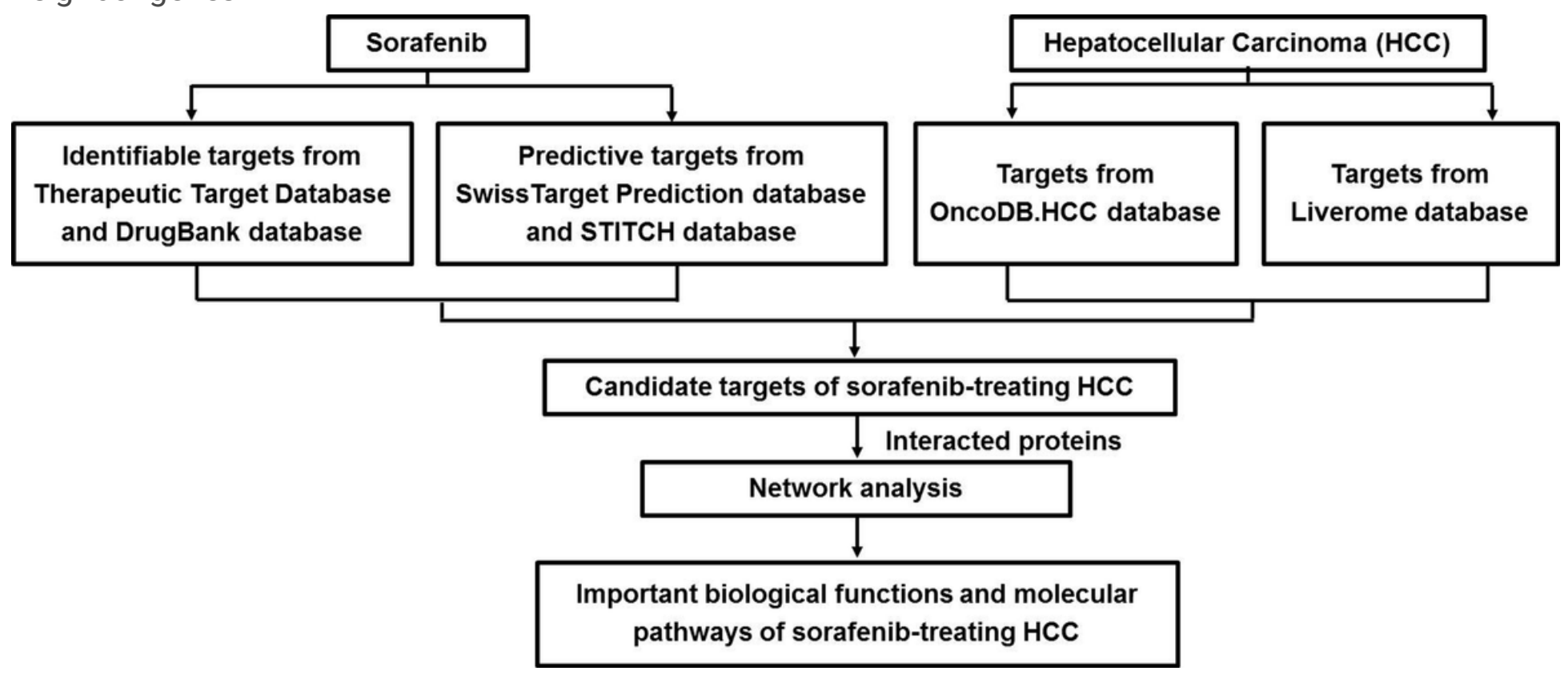

Figure 10

The flowchart of network pharmacology analysis of sorafenib administration for HCC treatment. 
a

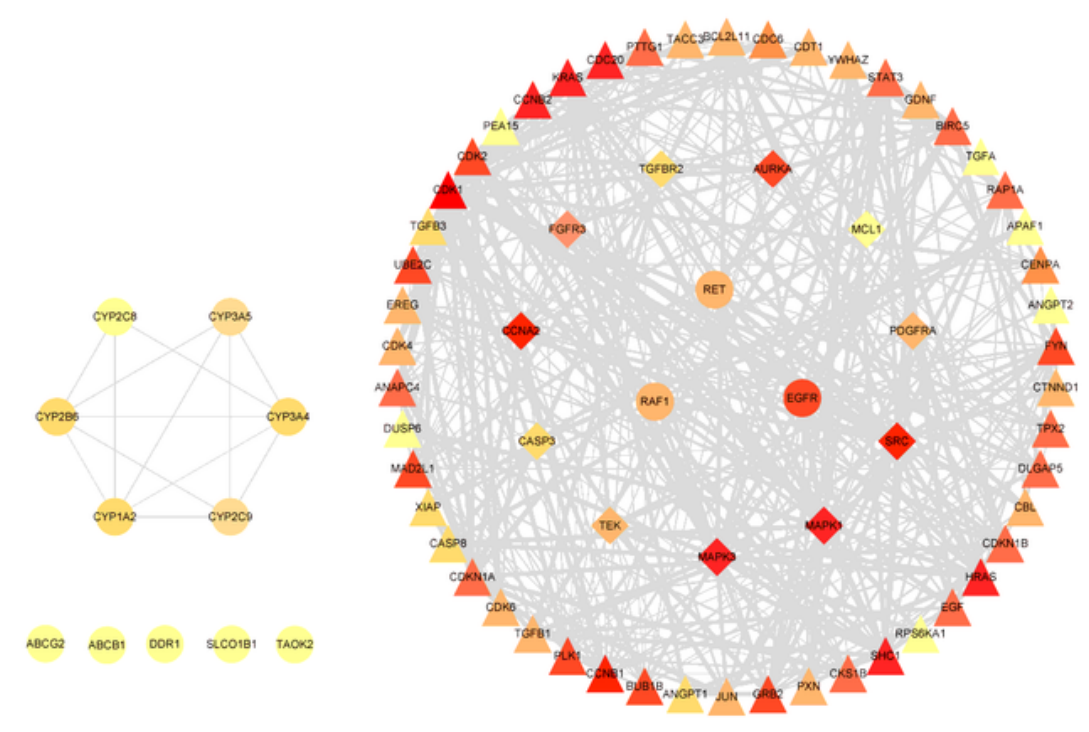

b

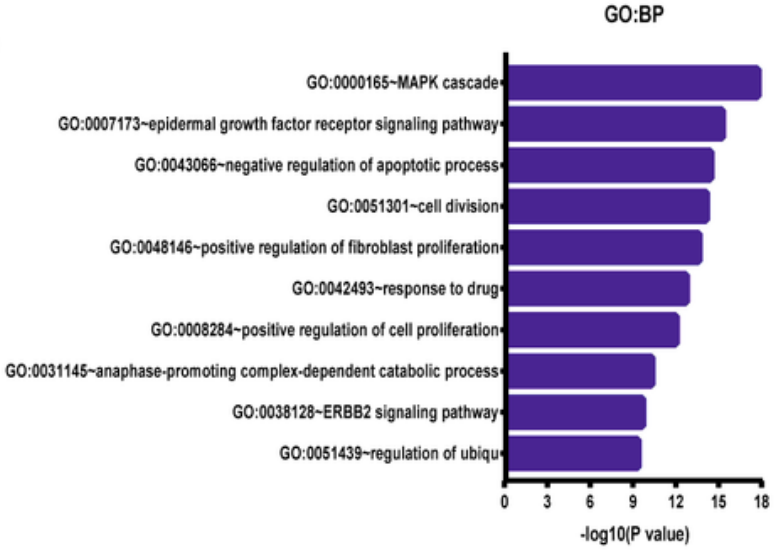

d

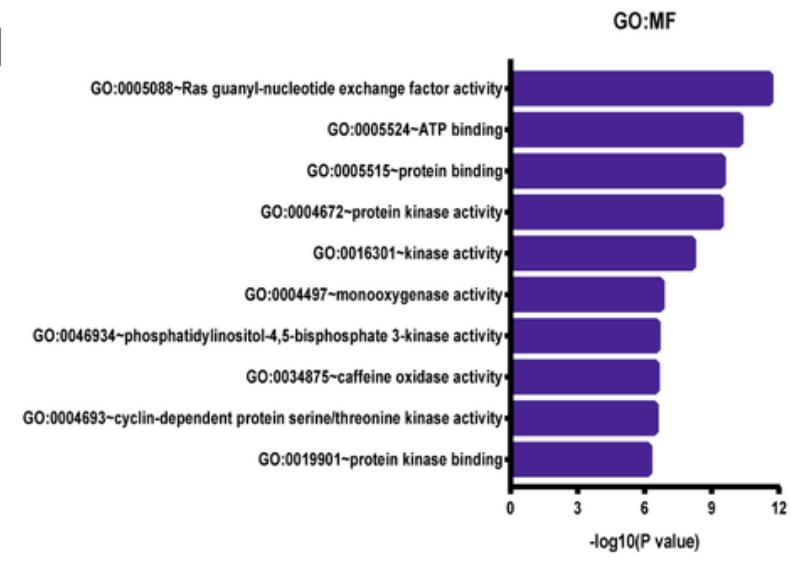

C

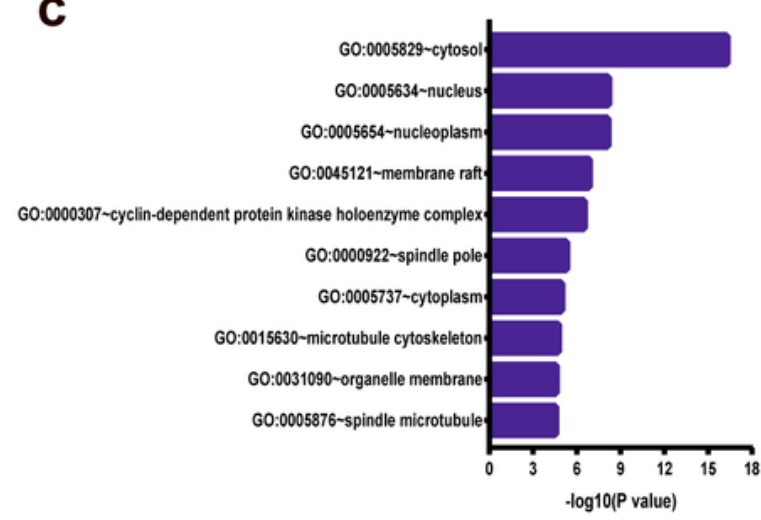

e

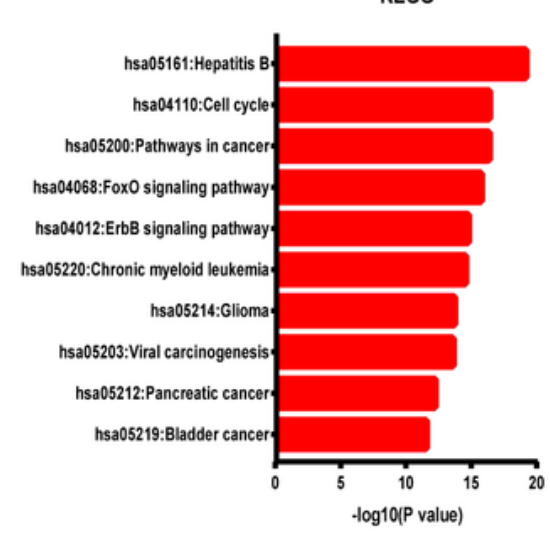

\section{Figure 11}

Protein-protein interaction (PPI) network (a), Gene ontology (b-d) and KEGG pathway enrichment (e) analysis of the therapy target genes of sorafenib on HCC. Ellipse represents identifiable targets, diamond corresponds to predictive targets, and triangle indicates other human targets.

\section{Supplementary Files}


This is a list of supplementary files associated with this preprint. Click to download.

- Supplementarymaterials.rar 